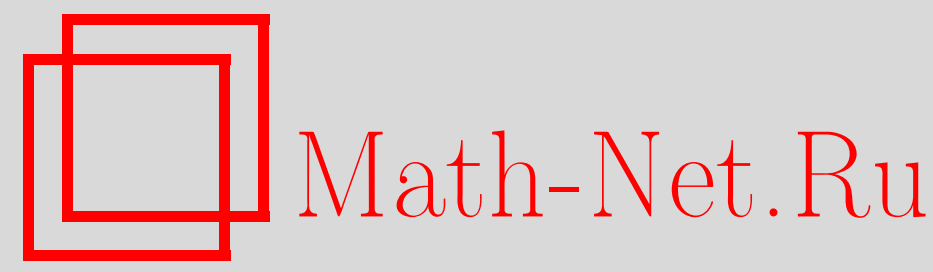

С. П. Суетин, Об асимптотических свойствах полюсов диагональных аппроксимаций Паде для некоторых обобщений марковских функций, Матем. сб., 2002, том 193, номер 12, 105-133

DOI: https://doi.org/10.4213/sm701

Использование Общероссийского математического портала Math-Net.Ru подразумевает, что вы прочитали и согласны с пользовательским соглашением

http://www. mathnet.ru/rus/agreement

Параметры загрузки:

IP : 34.227 .88 .159

26 апреля 2023 г., 17:05:50 


\author{
С.П. Суетин
}

\title{
Об асимптотических свойствах полюсов диагональных аппроксимаций Паде для некоторых обобщений марковских функций
}

\begin{abstract}
В работе получена нелинейная система дифференциальных уравнений ("обобщенная система Дубровина”), с помощью которой описьпается поведение тех нулей полиномов, ортогональных на нескольких отрезках, которые располагаются в лакунах между этими отрезками. Показано, что та же самая система описьвает динамику подобных нулей и для ортогональных полиномов более общего вида - знаменателей диагональных аптроксимаций Паде функций, мероморфных на вещественной гиперэллиптической римановой поверхности.

На основе такого подхода получены некоторые уточнения результатов E.А. Рахманова о сходимости диагональных аппроксимаций Паде для рациональных возмущений марковских функций.

Библиографоия: 41 название.
\end{abstract}

\section{§1. Введение}

1. В классической теореме Маркова утверждается сходимость последовательности подходящих дробей для чебышёвской непрерывной дроби

$$
\frac{1}{A_{1} z+B_{1}}+\frac{1}{A_{2} z+B_{2}}+\cdots+\frac{1}{A_{n} z+B_{n}}+\cdots
$$

построенной непосредственно по лорановским коэффициентам $s_{k}, k=0,1, \ldots$, разложения в ряд в бесконечно удаленной точке $z=\infty$ функции вида

$$
\widehat{\mu}(z):=\int_{E} \frac{d \mu(x)}{z-x}
$$

где $\mu$-положительная мера с носителем $E \Subset \mathbb{R}, s_{k}-$ ее моменты (для таких функций все $\left.A_{n} \neq 0\right)$. Функции вида (2) были введены П. Л. Чебышёвым [1] при изучении им свойств формальных разложений лорановских рядов в непрерывные дроби. Именно на этом пути Чебышёв открыл общие ортогональные многочлены, соответствующие произвольной положительной мере $\mu$ : такие многочлены естественньм образом возникают как знаменатели $Q_{n}$ для $n$-х подходящих дробей $P_{n} / Q_{n}$ непрерывной дроби (1). В теореме Маркова [2] речь идет о сходимости последовательности подходящих дробей $\left\{P_{n} / Q_{n}\right\} \kappa$ функции $\widehat{\mu}$ равномерно внутри

Работа вьполнена при финансовой поддержке Российского фонда фундаментальных исследований (грант № 02-01-00564), Программы подлержки ведущих научных школ России (грант № 00-15-96132) и ффонда INTAS (грант № 2000-272). 
(т.е. на компактных подмножествах) области $\overline{\mathbb{C}} \backslash[a, b]$, әде $[a, b]=\widehat{E}-$ выпуклая оболочка $E$. Хорошо известно, что $\widehat{E}$ нельзя заменить на $E$ : еше Марков [3] отмечал, что в случае, когда $E$ состоит из двух отрезков, в лакуне между ними может располагаться нуль ортогонального полинома $Q_{n}$, т.е. полюс $n$-й подходящей дроби. В “типичном” же случае множество предельных точек последовательности нулей полиномов $Q_{n}$ совпадает со всем отрезком $[a, b]=\widehat{E}$ (подробнее см. [4], [5]).

Подходящая дробь $P_{n} / Q_{n}$ обладает следуюшим замечательным свойством максимально возможного (по числу $2 n$ параметров) “касания" функции $\widehat{\mu}$ в точке $z=$ $\infty$ :

$$
\left(\widehat{\mu}-\frac{P_{n}}{Q_{n}}\right)(z)=O\left(\frac{1}{z^{2 n+1}}\right), \quad z \rightarrow \infty ;
$$

тем самьм, эта дробь есть в точности $n$-я диагональная аппроксимация Паде $[n / n]_{\widehat{\mu}}$ функции $\widehat{\mu}$ в точке $z=\infty$. Полиномы $P_{n}$ и $Q_{n}, \operatorname{deg} P_{n}=n-1, \operatorname{deg} Q_{n}=n$, полностью характеризуются (в классах полиномов степеней соответственно $\leqslant n-1$ и $\leqslant n$ и при подходяшей нормировке, например $\left.Q_{n}(0)=1\right)$ соотношением

$$
R_{n}(z):=\left(Q_{n} \widehat{\mu}-P_{n}\right)(z)=O\left(\frac{1}{z^{n+1}}\right), \quad z \rightarrow \infty
$$

Функция $R_{n}=R_{n}(z ; \widehat{\mu})$ в теории ортогональных полиномов называется функцией второго рода; в теории аппроксимаций Паде $R_{n}$ - это функиия остатка.

В настоящей работе изучаются диагональные аппроксимации Паде $[n / n]_{f}$ для функций вида

$$
f=\widehat{\mu}+r
$$

где $\widehat{\mu}$ - функция вида (2), $\mu$ - абсолютно непрерывная (вообще говоря, комплексная) мера из определенного класса (см. (5)), $r \in \mathbb{C}(z)$ - комплексная рациональная функция, голоморфная на $E, r(\infty)=0$. Тем самым, $f$ - “рациональное возмущение" $\widehat{\mu}$. Соответствующие полиномы $Q_{n}(z ; f)$ - знаменатели рациональной дроби $[n / n]_{f}$ - оказываются ортогональными относительно дискретного возмущения меры $\mu$, которое в случае, когда все полюсы $b_{j}$ функции $r$ простые, имеет вид $\mu+\sum_{j=1}^{m} \lambda_{j} \delta_{b_{j}}, b_{j}, \lambda_{j} \in \mathbb{C}$.

Первый результат о сходимости диагональных аппроксимаций Паде $[n / n]_{f}$ для функций вида (3) был получен А. А. Гончаром [6].

ТЕОРемА А. Пусть $\mu-$ положительная мера с носителем $E=[a, b] u$ $\mu^{\prime}=d \mu / d x>0$ почти всюдуна $E, r$ - комплексная рачиональная функиия, голоморфная на $E$ (т.е. $r \in \mathbb{C}(z) \cap \mathscr{H}(E)), r(\infty)=0$. Тогда диагональние аппроксимации Паде $[n / n]_{f}$ равномерно сходятся ${ }^{1} \kappa f=\widehat{\mu}+r$ внутри области $D=\overline{\mathbb{C}} \backslash E$.

Тем самьм, каждый полюс $f$ в $D$ притягивает ровно столько полюсов ${ }^{2}[n / n]_{f}$, какова его кратность.

Этот результат А.А. Гончара носит принципиальный характер: из него вытекает, что диагональные аппроксимации Паде, которые строятся непосредственно

\footnotetext{
${ }^{1}$ Здесь и всюду в дальнейшем, когда речь идет о мероморфных функциях, имеется в виду равномерная сходимость в сферической метрике.

${ }^{2}$ Как обычно, полюсы и нули функций считаются с учетом их кратностей.
} 
по лорановским коэффициентам ряда, в классе функций вида $f=\widehat{\mu}+r$ при указанных условиях эффективно решают задачу мероморфного продолэсения функции, заданной своим разложением в ряд: полюсы этих аппроксимаций локализуют отрезок $[a, b]$ и особые точки (полюсы и их кратности) функции $f$ в $\mathbb{C} \backslash[a, b]$, а сами аппроксимации представляют собой конструкцию, реализующую продолжение ряда в $D=\overline{\mathbb{C}} \backslash[a, b]$. В частности, эти рациональные дроби восстанавливают дивизор полюсов функции $f$ вида (3) в области $D$. Как хорошо известно, это замечательное свойство аппроксимаций Паде основано на том, что все их nолюсы “свободны” и определяются только условием максимального касания в бесконечно удаленной точке.

Естественным продолжением исследований А.А. Гончара стала работа Е.А. Рахманова [7], где был рассмотрен случай, когда носитель меры имеет лакуны. Точнее, в [7] получены следующие результаты.

ТЕОРемА В. Пусть носитель Е меры $\mu$ состоит из нескольких отрезков, а сама мера имеет вид $d \mu(x)=s(x) d x$, где весовая функиия $s(x) \geqslant 0$ на $E$ и удовлетворяет условию Сегё; $r \in \mathbb{C}(z) \cap \mathscr{H}(E), r(\infty)=0$. Тогда диагональние аппроксимации Паде $[n / n]_{f}$ сходятся по (логарифмической) емкости к $f=\widehat{\mu}+r$ внутри $D=\overline{\mathbb{C}} \backslash E$.

Из этого результата вытекает, что каждый полюс $f$ в $\mathbb{C} \backslash E$ притягивает no крайней мере столько полюсов $[n / n]_{f}$, какова его кратность. Метод же доказательства позволяет сделать следующий важный вывод: единственным nрепятствием к равномерной сходимости всей последовательности $\left\{[n / n]_{f}\right\}_{n \in \mathbb{N}}$ является “неопределенное" поведение некоторых из полюсов $[n / n]_{f}$ (нулей $\left.Q_{n}(z ; f)\right)$; число таких полюсов с неопределенным поведением не превышает числа лакун. В терминах гармонических мер, соответствующих отрезкам носителя меры, Рахманов получил в [7] систему уравнений, описывающих динамику таких нулей полиномов $Q_{n}(z ; f)$ в зависимости от $n$.

В случае одной лакуны единственное уравнение задает некоторую кривую $\gamma$, соединяюшую оба отрезка. В этом случае возможен лишь один нуль $\widetilde{z}_{n}$ полинома $Q_{n}(z ; f)$ с неопределенным поведением, который фактически "блуждает" около этой кривой: все предельные точки последовательности $\left\{\widetilde{z}_{n}\right\}$ лежат на $\gamma$. В “типичной" ситуации каждая точка $\gamma$ - предельная точка последовательнос$m u\left\{\widetilde{z}_{n}\right\}$.

Теорема С. Пусть Е есть обвединение двух непересекающихся отрезков вещественной оси, $E=E_{1} \cup E_{2}$, мера $d \mu(x)=s(x) d x$, где весовая функция $s(x) \geqslant 0$ на $E$ и удовлетворяет условию Сегё; $f=\widehat{\mu}+r$, где $r \in \mathbb{C}(z) \cap \mathscr{H}(E)$, $r(\infty)=0$. Тогда существует открытая аналитическая дуга $\gamma \subset D$, концы которой принадлежст отрезкам $E_{1}$ и $E_{2}$, такая, что

$$
[n / n]_{f} \rightrightarrows f \quad \text { внутри } \quad D \backslash \gamma
$$

Если гармоническая мера $\omega_{1}(\infty)=\omega\left(E_{1}, \infty ; D\right)$ - иррациональное число, то полюсы $[n / n]_{f}$ всюду плотны на $\gamma ;$ в противном случае существует конечное множество точек $Z \subset \gamma$ такое, что

$$
[n / n]_{f} \rightrightarrows f \quad \text { внутри } \quad D \backslash Z
$$


Дуга $\gamma$ зависит только от множества $E=E_{1} \cup E_{2}$ и полюсов функиии $r$ (и не зависит от меры $\mu$ и вычетов функции $r$ в полюсах).

Дуга $\gamma$, вообще говоря, лежит в $\overline{\mathbb{C}} \backslash \widehat{E}$ и есть не что иное, как “половина" ортогональной траектории квадратичного дифференциала на двулистной эллиптической римановой поверхности (подробнее см. $\S 4)$. Отметим, что если в условиях этой теоремы $r \equiv 0$, то дуга $\gamma-$ (конечная) лакуна между отрезками $E_{1}$ и $E_{2}$. Как хорошо известно, если в (3) $d \mu>0$, a $r \equiv 0$, то в случае произвольного числа лакун "блуждающие" 3 нули ортогональных полиномов могут располагаться только в лакунах между отрезками. Полученные в [4] (см. также [5; $§ 4$, п. 4.2]) в терминах гармонических мер $\omega_{j}(\infty)=\omega\left(E_{j}, \infty ; D\right)$ составляющих $E$ отрезков $E_{j}$ $(j=1, \ldots, g+1$, где $g \in \mathbb{N}$ - число лакун) уравнения в совокупности с развитым там же методом, основанным на решении специальной краевой задачи Римана и сингулярного интегрального уравнения, фактически означают, что в типичном случае блужсдающие нули всюду плотны в лакунах (см. также [8], где близкие результаты доказаны с помощью классического аппроксимационного метода Бернштейна-Сегё). Тем самым, в этом частном случае аналог теоремы С справедлив для произвольного $g \in \mathbb{N}$, а кривые $\gamma_{j}-$ лакуны между отрезками.

В общем случае для произвольного числа лакун непосредственно из уравнений Рахманова не удалось извлечь явного описания динамики блуждающих нулей полиномов $Q_{n}(z ; f)$. В частности, оставался открытым вопрос о том, как устроено множество предельных точек полюсов аппроксимаций Паде, возможно ли в общем случае такое же простое геометрическое описание этого множества, как полученное Рахмановьм при $g=1$.

Оказалось, что в обшем случае множество предельных точек нулей ортогональных полиномов $Q_{n}(z ; f)$ устроено довольно сложно и не имеет такого прозрачного геометрического описания, как при $g=1$. Соответствующий результат отрицательного характера установлен в настоящей работе для случая $g=2$ (см. 55$)$ : показано, что множество предельных точек, вообще говоря, “двумерно". Однако при некоторых дополнительных условиях это множество допускает аналитическое описание в терминах некоторой нелинейной системь дифференииальных уравнений ("обобшенной системы Дубровина"). Точнее, в настоящей работе получены интегрируемые дифференциальные уравнения (эквивалентные уравнениям Рахманова), описывающие (см. теоремы 1 и 3) динамику блуждающих нулей полиномов $Q_{n}(z ; f)$ в случае произвольного (конечного) числа лакун, а также динамику “свободных" (т.е. дополнительных к $(n+1)$-му нулю в бесконечно удаленной точке) нулей функций остатка $R_{n}(z ; f)$. В частности, для случая одной лакуны (т.е. при $g=1$ ) показано, что результат Рахманова сохраняется, если положить $r \equiv 0$, но зато допустить комплексные меры $\mu$ и, тем самым, ограничиться голоморфными функциями. При этом и в том и в другом случае соответствующая кривая $\gamma$-часть ортогональной траектории некоторого квадратичного дифференциала. В случае же произвольного числа лакун естественным образом возникают некоторые “семейства" кривых - обобщения траекторий квадратичных дифференциалов на римановых поверхностях, - которые содержат интересующие нас траектории. Структуру таких множеств удается пока изучить лишш при некоторых дополнительных ограничениях на "начальные условия", а также для случая $g=2$. Но уже полученных результатов оказывается достаточным для того, что-

\footnotetext{
${ }^{3}$ По поводу этого термина см. ниже п. 2.
} 
бы сделать принципиальньй вывод: множество предельных точек, вообще говоря, двумерно, т.е. аналог теоремы $\mathrm{C}$ не имеет места.

Отметим, что подобные исследования тесно связаны с результатами обратного характера, в которых на основании той или иной информации об асимптотическом поведении полюсов диагональных аппроксимаций Паде требуется сделать определенные выводы о свойствах аналитического продолжения заданной функции. Первым результатом в этом направлении для диагональных аппроксимаций Паде является теорема Гончара [9]. Наиболее общий прямой результат о сходимости таких аппроксимаций - это теорема Шталя [10], из которой, в частности, вытекает сходимость по емкости диагональных аппроксимаций Паде для произвольной $а л-$ гебраической функции вне некоторой “симметричной” системы разрезов $S$, в дополнении к которой выделяется однозначная ветвь исходного лорановского разложения. Непосредственно из этого результата Шталя вытекает, что каждьй полюс $f$ в $D=\overline{\mathbb{C}} \backslash S$ притягивает по крайней мере столько полюсов $[n / n]_{f}$, какова его кратность. Вопрос о том, справедлив ли в этом утверждении "знак равенства", т.е. восстанавливает ли диагональ Паде алгебраической функции дивизор ееполюсов в $D$, и составляет фактически задачу обратного характера, в общем случае до сих пор нерешенную. Некоторые результаты в этом направлении получены недавно Шталем [11] и автором [4] для гиперэллиптических функций и их естественных обобщений в ситуации “общего положения". При этом и в [11], и в [4] эти результаты получены как следствие доказательства (в "обшем положении") хорошо известной гипотезы Бейкера-Гаммеля-У иллса (см. [12], а также [11] и [13]). После того как Буслаев [14], [15] предъявил гиперэллиптическую функцию рода $g=2$, опровергающую эту гипотезу (для случая, когда $S \not \subset \mathbb{R}$ и состоит из трех компонент равной гармонической меры), стало ясно, что для исследования задач обратного характера в общем случае надо искать новые подходы. Возможно, предложенные в настояшей работе идеи окажутся в дальнейшем полезными именно с такой точки зрения. В наибольшей степени здесь удалось исследовать случай $g=2$, хотя даже и в этом случае обратную задачу решить пока не удается (эллиптическому случаю $g=1$ предполагается посвятить отдельную работу).

2. Всюду в дальнейшем предполагается, что множество $E \Subset \mathbb{R}$ состоит из конечного числа (непересекающихся) отрезков, $g \in \mathbb{N}$ - число лакун в $E$. Пусть $E_{j}=\left[e_{2 j-1}, e_{2 j}\right], j=1, \ldots, g+1,-$ составляющие множество $E$ отрезки, $h(z)=$ $z^{2 g+2}+\cdots \in \mathbb{R}[z]-$ полином степени $2 g+2$ с нулями в точках $e_{j}$ - концах составляющих отрезков. Через $E_{j}^{\prime}=\left(e_{2 j}, e_{2 j+1}\right), j=1, \ldots, g$, обозначим разделяющие $E$ (открытые) лакуны, $E_{g+1}^{\prime}$ - “внешняя" лакуна, содержашая бесконечно удаленную точку.

Предполагается, что $\mu$ - абсолютно непрерьвная комплексная мера на $E$ следующего вида: $d \mu(x)=\rho(x) d x /(\pi \sqrt{-h(x+i 0)})$, где под $\sqrt{-h(x+i 0)}, x \in E$, понимаются "верхние" граничные значения на $E$ той однозначной в $D$ ветви корня, которая определяется условием $\sqrt{h(z)} / z^{g+1} \rightarrow 1$ при $z \rightarrow \infty, z \in D$; комплекснозначная функция $\rho(x) \neq 0$ и вещественно аналитична на $E$ (как функция вещественной переменной $x \in E$ ). Тем самым, $\widehat{\mu}$ допускает следующее представление:

$$
\widehat{\mu}(z)=\frac{1}{\pi} \int_{E} \frac{\rho(x)}{\sqrt{-h(x+i 0)}} \frac{d x}{z-x}=\frac{1}{\pi i} \int_{E} \frac{\rho(x)}{\sqrt{h(x+i 0)}} \frac{d x}{x-z}=: \widehat{\rho}(z ; h) .
$$

Функцию $\rho$ в дальнейшем будем называть допустимылм весом; в частном случае, когда $\rho(x)$ - рациональная функция переменной $x(\rho \in \mathbb{C}(x))$, не имеющая на $E$ ни 
нулей, ни полюсов, $\widehat{\rho}(\cdot ; h)$ - гиперэллиптическая функция, определенная на гиперэллиптической римановой поверхности $\mathfrak{R}$ рода $g$, заданной уравнением $w^{2}=h(z)$ :

$$
\widehat{\rho}(z ; h)=r_{1}(z)+r_{2}(z) w
$$

где $r_{1}, r_{2} \in \mathbb{C}(z)$; тем самым, $\widehat{\rho}(\cdot ; h) \in \mathbb{C}(z, w)$. В дальнейшем, допуская некоторую вольность в обозначениях, при записи функции вида (5) ссылку на $h$ будем, как правило, опускать и писать $\widehat{\rho}(z)$ или просто $\widehat{\rho}$. Наконец, отметим, что в соответствии с выбранной нами ветвью корня марковскому случаю положительной меры $\mu$ соответствует такой допустимый вес $\rho$, для которого $i \rho / \sqrt{h}>0$ на $E$. Такой вес $\rho$ будем называть марковским. В классическом случае $g=0$ и $E=[-1,1]$ полиномы $Q_{n}$ ортогональны на $E$ относительно веса $\rho(x) / \sqrt{1-x^{2}}$, где $\rho>0$ - так называемый тригонометрический вес [16], а $d \mu(x)=\rho(x) d x /\left(\pi \sqrt{1-x^{2}}\right)$ и

$$
\widehat{\rho}(z)=\frac{1}{\pi} \int_{-1}^{1} \frac{\rho(x)}{\sqrt{1-x^{2}}} \frac{d x}{z-x} .
$$

Рассмотрим следующую систему нелинейных дифференциальных уравнений относительно неупорядоченного набора точек $\mathbf{z}_{1}(t), \ldots, \mathbf{z}_{g}(t), t \in \mathbb{R}_{+}$, на римановой поверхности $\mathfrak{R}$ (т.е. относительно дивизора $\left.d(t)=\mathbf{z}_{1}(t)+\cdots+\mathbf{z}_{g}(t)\right)$ :

$$
\dot{z}_{k}=-\frac{w\left(\mathbf{z}_{k}\right)}{\prod_{j \neq k}\left(z_{k}-z_{j}\right)} \int_{e_{2 g+2}}^{\infty} \frac{\prod_{j \neq k}\left(x-z_{j}\right)}{w(x)} d x, \quad k=1, \ldots, g,
$$

где $\dot{z}_{k}=d z_{k} / d t, \mathbf{z}=(z, \pm \sqrt{h(z)}), w(\mathbf{z})= \pm \sqrt{h(z)}, e_{2 g+2}-$ крайняя правая точка множества $E$ (предполагается, что путь интегрирования в $(*)$ - часть вещественной оси $\left[e_{2 g+2},+\infty\right)$; подробнее по поводу обозначений см. $\left.\S 2\right)$.

Подмножество поверхности $\mathfrak{R}$, которое заполняют точки $\mathbf{z}_{j}(t)$, составляющие дивизор $d(t)$ и удовлетворяющие $(*)$, зависит, вообще говоря, от начальных условий $\mathbf{z}_{j}^{0}=\mathbf{z}_{j}(0)$. В частности, если все $z_{j}(0)$ лежат в лакунах между отрезками, то и все $z_{j}(t)$ принадлежат этим же лакунам. Начальные условия всегда можно задать так, что соответствующий дивизор $d^{0}=\mathbf{z}_{1}^{0}+\cdots+\mathbf{z}_{g}^{0}$ неспециальньй. В дальнейшем мы будем фактически предполагать, что дивизор начальных условий $d^{0}$ таков, что и при каждом $t \in \mathbb{R}_{+}$дивизор

$$
d(t)=\mathbf{z}_{1}(t)+\cdots+\mathbf{z}_{g}(t)-\text { неспециальньй, где } d(0)=d^{0} ;
$$

здесь $\mathbf{z}_{j}(t)$ - решения (*). Такие начальные условия будем называть правильными (соответственно, дивизор $d^{0}-$ правильным; см. $\S 2$, п. 3).

Обсудим это ограничение: при $g=1$ (6) выполнено всегда; при $g=2$, как будет показано ниже в $\S 5$, условие (6) почти всегда выполняется и может быть задано явно в терминах $x$-координат дивизора начальных условий $d^{0}$. Наконец, при произвольном $g \geqslant 3$ выбор таких начальных условий, что $z_{j}^{0} \in E_{j}^{\prime}$, приводит к тому, что и при всех $t \in \mathbb{R}_{+}$точки $z_{j}(t) \in E_{j}^{\prime}$. Следовательно, соответствующий дивизор $d(t)$ всегда неспециальный. Пока не удается выяснить, сохраняется ли для $g \geqslant 3$ свойство (6) при каких-то еще начальных условиях, кроме указанных (подробнее см. $\S 2$ ). 
Обозначим через $D^{(1)}$ “первый” (открытый) лист римановой поверхности $\Re$, состоящий из точек $\mathbf{z}=(z, \sqrt{h(z)}), z \notin E$, а через $D^{(2)}$ - “второй" лист: $\mathbf{z} \in D^{(2)}$, если $\mathbf{z}=(z,-\sqrt{h(z)})$; в соответствии с таким определением для точек $\mathbf{z} \in \mathfrak{R}$ мы будем использовать обозначения $z^{(1)}, z^{(2)}$ с явным указанием листа или обозначение $\mathbf{z}=(z, \pm)$. Имеем $\operatorname{pr} D^{(1)}=\operatorname{pr} D^{(2)}=D$, где $D=\overline{\mathbb{C}} \backslash E$, a pr - каноническая проекция $\mathfrak{R}$ на $\overline{\mathbb{C}}: \operatorname{pr}(z, \pm)=z$. Тем самым, $\mathfrak{R}=D^{(1)} \cup L \cup D^{(2)}$, где $L=E^{+} \cup E^{-}$.

Пусть $\boldsymbol{\Gamma}_{j}$ - траектория, которую описьвает на $\mathfrak{R} j$-я точка $\mathbf{z}_{j}(t)$ с начальными условиями $\mathbf{z}_{j}(0)=\mathbf{z}_{j}^{0}$ под “действием” динамической системы $(*)$. Тогда $\boldsymbol{\Gamma}_{j}-$ (необязательно замкнутая) аналитическая кривая на $\mathfrak{R}$, одна часть $\Gamma_{j}^{(1)}$ которой лежит на первом листе, а другая $\Gamma_{j}^{(2)}$ - на втором листе. Проекция $\Gamma_{j}$ кривой $\boldsymbol{\Gamma}_{j}$ на $\overline{\mathbb{C}}$ - вообще говоря, незамкнутая кривая. Расположение $\boldsymbol{\Gamma}_{j}$ на $\mathfrak{R}$ зависит от начальных условий $\mathbf{z}_{j}^{0}$; в частности, если все $z_{j}(0)$ лежат в лакунах между отрезками, то $\Gamma_{j}$ совпадают с замьканиями этих лакун, т.е. $\Gamma_{j}=\left[e_{2 j}, e_{2 j+1}\right]$.

Одним из основных результатов настояшей работы является

ТЕОРЕМА 1. Пусть допустимый комплекснозначный вес $\rho(x) \neq 0$ имеет вид $\rho=1 / p$, где $p \in \mathbb{C}[z](p(x) \neq 0$ на $E), f=\widehat{\rho}, d^{0}$ - начальнье условия, соответствующие функиии $\rho$, и дивизор $d^{0}$ правильный.

1) Если для некоторой последовательности $\Lambda_{1} \subset \mathbb{N}$ и некоторого $j \in$ $\{1, \ldots, g\}$ все точки $\mathbf{z}_{j}(n)=z_{j}^{(1)}(n) \in D^{(1)}$ при $n \in \Lambda_{1}$, то для $n \in \Lambda_{1}$ функиия остатка $R_{n}(z ; \widehat{\rho})$ имеет нуль в точке $z_{j}(n) \in D$.

2) Если для некоторой последовательности $\Lambda_{2} \subset \mathbb{N}$ и некоторого $j \in$ $\{1, \ldots, g\}$ все точки $\mathbf{z}_{j}(n)=z_{j}^{(2)}(n) \in D^{(2)}$ при $n \in \Lambda_{2}$, то для $n \in \Lambda_{2}$ полином $Q_{n}(z ; \widehat{\rho})$ имеет нуль $\widetilde{z}_{n}$, который бесконечно близок $к$ точке $z_{j}(n) \in D$ при $n \rightarrow \infty$.

Указанным закономерностям подчиняется асимптотическое поведение всех нулей функиий $R_{n}$ и $Q_{n}$, имеющих предельные точки в $D \backslash\{\infty\}$.

Таким образом, в условиях теоремы 1 поведение всех блуждающих нулей пары $\left(Q_{n}, R_{n}\right)$ подчиняется динамической системе $(*)$. Как уже отмечалось, при $\rho=1 / p$ функция $f=\widehat{\rho}$ гиперэллиптична на римановой поверхности $\Re$. Тем самњм, в условиях теоремы 1 удается свести вопрос о сходимости последовательности $[n / n]_{f}$ для гиперэллиптических функций вида $f=\widehat{1 / p}$ к вопросу о строении множества предельных точек тех решений системы $(*)$, которые соответствуют дискретным значениям $t=n$.

В следующей теореме устанавливаются некоторые свойства системы (*).

ТеОрема 2. Динамическая система (*):

1) интегрируется в явном виде преобразованием Абеля; решения системь - условно периодические функции переменной $t$ с периодами $\omega_{1}(\infty), \ldots, \omega_{g}(\infty)$;

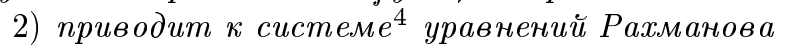

$$
\sum_{j=1}^{g} \varepsilon_{j} \omega_{k}\left(z_{j}\right)=(g-2 t) \omega_{k}(\infty)-2 \rho_{k} \quad(\bmod 2), \quad k=1, \ldots, g,
$$

описывающей движение дивизора $d(t)=\mathbf{z}_{1}(t)+\cdots+\mathbf{z}_{g}(t)$ по $\boldsymbol{\Gamma}_{1} \times \cdots \times \boldsymbol{\Gamma}_{g}$, әде $\varepsilon_{j}= \pm 1$ в зависимости от $\mathbf{z}_{j}=\left(z_{j}, \pm 1\right.$ ) (подробнее см. $\S 3$, п. 4 );

\footnotetext{
${ }^{4}$ Приводимые ниже уравнения составляют лишь часть уравнений Рахманова.
} 
3) в пределе при $e_{2 g+2} \rightarrow \infty$ переходит в систему уравнений Дубровина

$$
\dot{z}_{k}= \pm \frac{2 \sqrt{-h\left(z_{k}\right)}}{\prod_{j \neq k}\left(z_{k}-z_{j}\right)}, \quad k=1, \ldots, g
$$

описывающую динамику нулей конечнозонных решений уравнения Шрёдингеpa.

В “типичном" случае (а именно если $\omega_{1}(\infty), \ldots, \omega_{g+1}(\infty)$ рационально независимы ) множество $\left(\mathbf{z}_{1}(n), \ldots, \mathbf{z}_{g}(n)\right)$ всюду плотно на $\boldsymbol{\Gamma}_{1} \times \cdots \times \boldsymbol{\Gamma}_{g}$. Тем самым, множество блуждающих нулей $R_{n}(z ; f)$ всюду плотно на $\operatorname{pr} \Gamma^{(1)}$, а множество блуждающих нулей $Q_{n}(z ; f)$ - на $\operatorname{pr} \Gamma^{(2)}$, где $\boldsymbol{\Gamma}=\bigcup_{j=1}^{g} \boldsymbol{\Gamma}_{j}$.

Таким образом, переход от вешественнозначных весов к комплекснозначнњм приводит к тому же принципиально новому эффекту, который в эллиптическом случае $g=1$ был обнаружен Рахмановьм при рациональном возмушении марковской функции: блуждающие нули ортогональных многочленов сходят с лакун и располагаются около некоторого множества в комплексной плоскости. Вполне вероятно, что изучение строения этого множества позволит в дальнейшем приблизиться к решению задач обратного характера.

Как хорошо известно [17], гармоническая мера $\omega(\ell, z ; G)$ дуги $\ell \subset \partial G$ в точке $z$ области $G \subset \mathbb{C}$ относительно границы $\partial G$ имеет следующий геометрический смысл: $\omega(\ell, z ; G)$ равна деленному на $2 \pi$ углу, под которым из точки $z \in G$ "видна" дуга $\ell$. Тем самьм, уравнения Рахманова (7) означают, что совокупность точек $\left(\mathbf{z}_{1}(n), \ldots, \mathbf{z}_{g}(n)\right)$ (т.е. дивизор $\left.d(t)=\mathbf{z}_{1}(n)+\cdots+\mathbf{z}_{g}(n)\right)$ движется по $\boldsymbol{\Gamma}_{1} \times \cdots \times \boldsymbol{\Gamma}_{g}$ с постоянной “угловой скоростью” (подробнее см. $\S 3$ ).

Система $(*)$ доставляет некоторую информацию о динамике блуждающих нулей полиномов $Q_{n}$ и функций остатка $R_{n}$ и в общем случае, т.е. для функций вида (3), в следующем смысле. Заданной паре $\rho, r$ соответствуют некоторые начальные условия $d^{0}$ (см. $\S 2$, п. 5); как и ранее, если дивизор $d^{0}$ правильный, то такое соответствие однозначно. Так будет, например, в случае, если вес $\rho$ марковский, а $r \in \mathbb{R}(z) \cap \mathscr{H}(E):$ тогда все $z_{j}(0)$ лежат в лакунах между отрезками.

Положим $\Gamma_{j}^{\circ}=\Gamma_{j} \backslash L$, где $L=E^{+} \cup E^{-}$.

ТЕОРЕма 3. Пусть $\rho(x) \neq 0$ - допустимыи (комплекснозначный вешественно

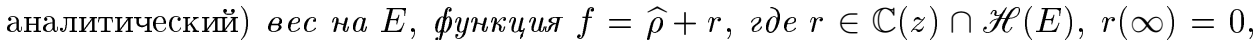
$d^{0}$ - начальнье условия, соответствующие паре функиий $(\rho, r)$, и дивизор $d^{0}$ правильный. Рассмотрим последовательность $\left\{\left(\mathbf{z}_{1}(n), \ldots, \mathbf{z}_{g}(n)\right)\right\} \subset \boldsymbol{\Gamma}_{1} \times$ $\cdots \times \Gamma_{g}, n \in \mathbb{N}$.

$1^{\circ}$. Если для некоторой последовательности $\Lambda_{1} \subset \mathbb{N}$ и некоторого $j \in$ $\{1, \ldots, g\}$ мнохсество $\left\{\left(\mathbf{z}_{1}(n), \ldots, \mathbf{z}_{j}(n), \ldots, \mathbf{z}_{g}(n)\right), n \in \Lambda_{1}\right\} \Subset \Gamma_{1}^{\circ} \times \cdots \times \Gamma_{j}^{(1)} \times$ $\cdots \times \Gamma_{g}^{\circ}$, то для $n \in \Lambda_{1}$ функция остатка $R_{n}$ имеет блуждающий нуль $\widehat{z}_{n}$, который бесконечно близок $\kappa z_{j}(n)$ при $n \rightarrow \infty$.

$2^{\circ}$. Если для некоторой последовательности $\Lambda_{2} \subset \mathbb{N}$ и некоторого $j \in$ $\{1, \ldots, g\}$ множество $\left\{\left(\mathbf{z}_{1}(n), \ldots, \mathbf{z}_{j}(n), \ldots, \mathbf{z}_{g}(n)\right), n \in \Lambda_{2}\right\} \Subset \Gamma_{1}^{\circ} \times \cdots \times \Gamma_{j}^{(2)} \times$ $\cdots \times \Gamma_{g}^{\circ}$, то для $n \in \Lambda_{2}$ полином $Q_{n}$ имеет блужсдающий нуль $\widetilde{z}_{n}$, который бесконечно близок $\kappa z_{j}(n)$ при $n \rightarrow \infty$.

Таким образом, при изучении явления блуждающих нулей естественно рассматривать пару функций $\left(Q_{n}, R_{n}\right), n \in \mathbb{N}$. 
Следствия к теоремам 1 и 3 приведены соответственно в $\S 3$ и $\S 4$.

Подчеркнем, что описание асимптотического поведения нулей $Q_{n}$ и $R_{n}$ с помощю системы $(*)$ оказалось возможным благодаря развитому в [4] (см. также [5]) подходу, в рамках которого асимптотические формулы для $Q_{n}$ и $R_{n}$ даются в терминах пары функций $\left(\varphi_{n}, \psi_{n}\right)$, решающих краевую задачу Римана на $L=E^{+} \cup E^{-}$ с заданной граничной функцией $\rho$, дивизоры (нулей и полюсов) которых удовлетворяют определенным требованиям. Точнее, при подходящей нормировке имеем: $Q_{n} \sim \varphi_{n}, R_{n} \sim \psi_{n} / w$ равномерно внутри $D$.

Наконец, обсудим терминологию. В теории аппроксимаций Паде те полюсы рациональных функций $[n / n]_{f}$ (а тем самым, и нули ортогональных полиномов $\left.Q_{n}(z ; f)\right)$, которые "не соответствуют" особенностям функции $f$, принято называть "spurious"5 (см. [13] и имеющиеся там дальнейшие ссылки). Однако фактически это лиш качественная характеристика ситуации, возникаюшей по некоторой подпоследовательности, а вовсе не конкретного объекта. Действительно, говорят [13], что по подпоследовательности $\Lambda$ имеется "spurious"-полюс (иначе говоря, “блуждающий” полюс) аппроксимачий Паде $[n / n]_{f}$, если при $n \in \Lambda$ по крайней мере $k$ полюсов рациональных функиий $[n / n]_{f}$ стремятся к некоторой точке $a \in D$, в то время как кратность полюса $f$ в этой точ$\kappa e \leqslant k-1$. При этом нет никакой возможности отличить "spurious"-полюсы от остальных. Введение нового термина не решает проблемы характеризации таких полюсов, но в отличие от "spurious" этот термин не носит негативного характера, а наоборот, подчеркивает некоторую закономерность в поведении этих “лишних" полюсов. Кроме того, установленная здесь при некоторых ограничениях тесная связь таких полюсов с решениями уравнения $(*)$ позволяет надеяться на то, что в будущем будет найдено их полное описание.

Результаты настоящей работы частично анонсированы в [18].

3. Как хорошо известно, впервые вопрос об асимптотических свойствах полиномов, ортогональных на нескольких отрезках вещественной прямой, был поставлен Н.И. Ахиезером в [19]. В этой работе, а также в [20] им фактически были получены явные (в терминах абелевых дифференциалов на гиперэллиптической римановой поверхности) выражения для функций второго рода $R_{n}$ и формулы асимптотики отношения таких функций, соответствующих двум различным весам вида “единица делить на полином" (ср. с условиями теоремы 1). Явные формулы для полиномов, ортогональных относительно таких специальных весов на отрезке $[-1,1]$, лежат в основе классического аппроксимационного метода Бернштейна-Сегё, используемого при доказательстве формулы сильной асимптотики ортогональных полиномов (в классическом случае $g=0$, а мера $\mu$ имеет вид $\left.d \mu(x)=\rho(x) d x /\left(\pi \sqrt{1-x^{2}}\right)\right)$. В [21] Н.И. Ахиезер фактически указал и метод изучения асимптотики при $n \rightarrow \infty$ тех нулей ортогональных многочленов, которые располагаются в лакунах между отрезками, - это проблема Якоби обрашения абелевых интегралов. Хотя в [19]-[21] рассматривается ортогональность на отрезках вещественной оси относительно марковских весов, предложенная Ахиезером методика оказалась по сушеству комплексной. Это наблюдение позволило Дж. Наттоллу [22], [23] существенно развить подход Ахиезера при изучении вопросов сходимости диагональных аппроксимаций Паде для произвольных гиперэллиптических функций и их естественных обобщений: знаменатели таких аппроксимаций -

\footnotetext{
${ }^{5}$ Общепринятого русского термина пока не существует.
} 
полиномы, неэрмитово ортогональные на некоторых дугах комплексной плоскости, обладающих определенньм свойством “симметрии".

Наиболее общие результаты о сильной асимптотике многочленов, әрмитово ортогональных на системе кривых и дуг в $\mathbb{C}$ относительно вешественного веса $\rho \geqslant 0$, удовлетворяющего условию Сегё, были получены Видомом [24]. Ответ дан им явно в терминах многозначных функций, определяемых геометрией заданной многосвязной области (это - гармонические меры, комплексные функции Грина, функции Неймана). Кроме того, в качестве параметров в ответе фигурируют решения проблемы обрашения Якоби. Эти параметры, как отметил Видом (см. [24; $\S 6$, c. 172]), могут быть стандартным образом описаны как нули $\theta$-функции Римана. В дальнейшем такая идеология, состоящая в редукции проблемы обрашения Якоби, соответствующей задаче об асимптотике ортогональных многочленов, к алгебраическим уравнениям, порождаемым $\theta$-функцией, была существенно развита А.И. Аптекаревым [25], а также, в случае полиномов, ортогональных на нескольких отрезках, в [26] и [27]. Вместе с тем, насколько нам известно, из полученных на этом пути асимптотических формул (см. [25; теорема 1], [26; теорема 1.34]) так и не было извлечено какого-либо конкретного описания динамики нулей ортогональных полиномов $Q_{n}$, расположенных в лакунах между отрезками.

В настоящей работе мы придерживаемся подхода Ахиезера-Наттолла к изучению динамики блуждающих нулей ортогональных полиномов, в основе которого непосредственное исследование проблемы Якоби обращения абелевых интегралов.

Отметим, что именно на таком пути в [4] (см. также [5]) доказано, что в $m u$ пичной ситуачии (точнее, если гармонические меры $\omega_{1}(\infty), \ldots, \omega_{g+1}(\infty)$ отрезков носителя рационально независимы) множество всех предельных точек нулей $Q_{n}$ совпадает с $\widehat{E}$. С другой стороны, по некоторой подпоследовательности $\Lambda$ нули ортогональных многочленов $Q_{n}$ не имеют предельных точек в лакунах между отрезками; тем самьм, по этой подпоследовательности диагональные аппроксимации Паде $\left\{[n / n]_{\widehat{\rho}}\right\}_{n \in \Lambda}$ сходятся к функции $\widehat{\rho}$ равномерно внутри $\overline{\mathbb{C}} \backslash E$, а не только внутри $\overline{\mathbb{C}} \backslash \widehat{E}$ (как в теореме Маркова). Аналогичньй результат о сушествовании (в типичной ситуации) равномерно сходящейся в $\overline{\mathbb{C}} \backslash E$ подпоследовательности аппроксимаций Паде получен в [4] и для функций вида $f=\widehat{\rho}+r$. Тем самым, показано, что в такой ситуации обратная задача имеет положительное решение. Наконец, хорошо известная работа Дюма [28] (см. также [29]) целиком основана на исследовании проблемы Якоби обрашения эллиптических интегралов, притом что в этом случае функция остатка $R_{n}$ - это эллиптическая функция, которая стандартным образом представляется в виде отношения (четырех) $\theta$-фуункций Якоби.

\section{§2. Основные определения и вспомогательные результаты}

1. Напомним, что множество $E \Subset \mathbb{R}$ состоит из конечного числа отрезков $E_{j}=$ $\left[e_{2 j-1}, e_{2 j}\right], j=1, \ldots, g+1, e_{1}<\cdots<e_{2 g+2} ; h(z)=z^{2 g+2}+\cdots-$ полином с нулями в точках $\left\{e_{k}\right\}$. Ветвь корня $\sqrt{h(z)}$ выбрана так, что $\sqrt{h(z)} / z^{g+1} \rightarrow 1$ при $z \rightarrow \infty$. Комплексная мера $\mu$ абсолютно непрерывна на $E$ и имеет вид $d \mu=$ $\rho(x) d x /(\pi \sqrt{-h(x+i 0)})$, где под $\sqrt{-h(x+i 0)}, x \in E$, понимаются "верхние" граничные значения на $E$ выбранной ветви корня, комплекснозначная функция $\rho(x) \neq 0$ и вещественно аналитична на $E$. Тем самњм, $\widehat{\mu}$ допускает представление $(5)$. 
Рассмотрим (вещественную) гиперэллиптическую риманову поверхность $\mathfrak{R}$ : $w^{2}=h(z)$ рода $g$, которая реализована как двулистное разветвленное накрытие римановой сферы $\overline{\mathbb{C}}$ таким образом, что переход с одного листа на другой осуществляется по верхнему $E_{j}^{+}$и нижнему $E_{j}^{-}$берегам отрезков $E_{j}$. Таким образом, отрезкам $E_{j}$ соответствуют на $\mathfrak{R}$ замкнутые аналитические (в комплексной структуре $\mathfrak{R})$ жордановы кривые $L_{j}, j=1, \ldots, g+1,-$ циклы на $\mathfrak{R}$; положим $L=\bigcup_{j=1}^{g+1} L_{j}$.

Приведем некоторые сведения о гиперэллиптических поверхностях, которые понадобятся нам в дальнейшем. В обозначениях и терминологии мы здесь придерживаемся в основном [30] (см. также [31]-[33]).

Каждой точке $z \neq e_{k}$ на $\overline{\mathbb{C}}$ соответствуют две точки $z^{(1)}=(z, \sqrt{h(z)})$ и $z^{(2)}=(z,-\sqrt{h(z)})$ на $\Re$. Пусть $D^{(1)}$ и $D^{(2)}$ - два (открытых) листа поверхности, склеенные по $L, \mathfrak{R}=D^{(1)} \cup L \cup D^{(2)}$; аналитическая функция $w=w(\mathbf{z})$, где $\mathbf{z}=z^{(1)}$ или $z^{(2)}$, однозначна на $\mathfrak{R}: w(\mathbf{z})=\sqrt{h(z)}$ при $\mathbf{z}=z^{(1)}$ и $w(\mathbf{z})=-\sqrt{h(z)}$ при $\mathbf{z}=z^{(2)}$. Для $\mathbf{z}=z^{(1)}$ будем часто писать просто $w(z)$ вместо $w(\mathbf{z})$; тем самьм, приобретает смысл и запись $w^{+}(x)=\sqrt{h(x+i 0)}, x \in E$. Каноническая проекция $\operatorname{pr}: \mathfrak{R} \rightarrow \overline{\mathbb{C}}$ определяется соотношением $\operatorname{pr}(\mathbf{z})=z$, в частности $\operatorname{pr} D^{(1)}=\operatorname{pr} D^{(2)}=D, \operatorname{pr} L=E$. Точки $z^{(1)}$ и $z^{(2)}$ на $\Re$ будем называть (взаимно) сопряжснными; иногда мы будем использовать обозначение $\mathbf{z}=(z, \pm)$.

Выберем ориентацию кривых $L_{j}=E_{j}^{+} \cup E_{j}^{-}$так, что при обходе по $L_{j}$ область $D^{(1)}$ остается слева, а $D^{(2)}$ - справа. Примем ориентированные кривые $L_{j}, j=1, \ldots, g$, за $a$-циклы $\mathbf{a}_{j}$ на $\mathfrak{R}$ и стандартным образом [30] дополним их $b$-циклами $\mathbf{b}_{j}$ до базиса гомологий $\left\{\mathbf{a}_{j}, \mathbf{b}_{j}\right\}_{j=1, \ldots, g}$ на $\mathfrak{R}$. Пусть $\left\{d \Omega_{k}\right\}-$ соответствующий $a$-нормированный базис голоморфных абелевых дифференциалов: $\oint_{\mathbf{a}_{j}} d \Omega_{k}=\delta_{k j}, k, j=1, \ldots, g$. Матрица $\mathbf{B}=\left\|B_{k j}\right\|_{k, j=1, \ldots, g}, B_{k j}=\oint_{\mathbf{b}_{j}} d \Omega_{k}-$ b-периоды базисных дифференциалов, является матрицей Римана: она симметрична, $B_{k j}=B_{j k}$, а ее мнимая часть положительно определена, $\left\|\operatorname{Im} B_{j k}\right\|>0$ (отметим, что в нашем случае все $\left.B_{j k} \in i \mathbb{R}\right)$. Функции $\Omega_{k}(\mathbf{z})=\int_{e_{2 g+2}}^{\mathbf{z}} d \Omega_{k}$ определены на $\mathfrak{R}$ однозначно по модулю их $a$ - и $b$-периодов; функции $\Omega_{k}^{\prime}(\mathbf{z})=p_{k}(z) / w(\mathbf{z})-$ однозначные мероморфные функции на $\mathfrak{R}, p_{k} \in i \mathbb{R}[z], \operatorname{deg} p_{k}=g$.

Если в $D^{(1)}$ задана функция $F(\mathbf{z})$, то под $F^{+}(\boldsymbol{\zeta})$ понимаются ее предельные значения при $\mathbf{z} \rightarrow \boldsymbol{\zeta} \in L, \mathbf{z} \in D^{(1)}$, если они существуют; аналогичньй смысл придается и $F^{-}(\boldsymbol{\zeta})$.

Фиксируем стандартньй базис $\mathbf{e}_{1}, \mathbf{e}_{2}, \ldots, \mathbf{e}_{g}$ в $\mathbb{R}^{g}:\left(\mathbf{e}_{k}\right)_{j}=\delta_{k j}$. Тогда векторы $\mathbf{e}_{1}, \ldots, \mathbf{e}_{g}, \mathbf{B e}_{1}, \ldots, \mathbf{B e}_{g}$ линейно независимы над $\mathbb{R}$ и образуют базис в $\mathbb{C}^{g}$. Факторпространство $\mathbb{C}^{g} /\{\mathrm{N}+\mathrm{BM}\}$ по целочисленной решетке, $\mathrm{N}, \mathrm{M} \in \mathbb{Z}^{g}$, является $2 g$-мерным вещественным тором $\mathbb{T}^{2 g}$ и называется многообразием Якоби Јас $\mathfrak{R}$ римановой поверхности $\mathfrak{R}$. Любой вектор $\mathbf{v} \in \mathbb{C}^{g}$ однозначно представим в виде $\mathbf{v}=\mathbf{x}+\mathbf{B y}+\mathrm{N}+\mathbf{B M}=\mathbf{x}+\mathbf{B y}(\bmod$ периодов $), 0 \leqslant(\mathbf{x})_{j},(\mathbf{y})_{j}<1$, $\mathrm{N}, \mathrm{M} \in \mathbb{Z}^{g}$; иногда для краткости знак сравнения по модулю периодов дифференциалов $\left\{d \Omega_{k}\right\}$ мы будем опускать и писать просто $\mathbf{v} \equiv \mathbf{x}+\mathbf{B y . ~ В е ш е с т в е н н ы е ~}$ числа $x_{j}=(\mathbf{x})_{j}, y_{j}=(\mathbf{y})_{j} \in[0,1), j=1, \ldots, g$, будем называть соответственно $x$ - и $y$-координатами вектора $\mathbf{v}$ в многообразии Якоби (точнее, класса $[\mathbf{v}]$ всех эквивалентных v векторов). 
Пусть $\mathbf{z}_{0}$ - некоторая фиксированная точка ${ }^{6}$ римановой поверхности $\mathfrak{R}$. Вектор-функция $A(\mathbf{z})=\left(\Omega_{1}(\mathbf{z}), \ldots, \Omega_{g}(\mathbf{z})\right)$ с координатами

$$
\Omega_{j}(\mathbf{z})=\int_{\mathbf{z}_{0}}^{\mathbf{z}} d \Omega_{j} \quad(\bmod \text { периодов }), \quad j=1, \ldots, g,
$$

определяет отображение Абеля $A: \mathfrak{R} \rightarrow$ Jас $\mathfrak{R}$ (подразумевается, что путь интегрирования - один и тот же для всех $j$ ). Неупорядоченные наборы точек $\left(\mathbf{z}_{1}, \ldots, \mathbf{z}_{g}\right)$, $\mathbf{z}_{j} \in \mathfrak{R}$, образуют $g$-ю симметрическую степень $S^{g} \mathfrak{R}$ римановой поверхности $\mathfrak{R}$. Вектор-функция $A\left(\mathbf{z}_{1}, \ldots, \mathbf{z}_{g}\right)=\left(A_{1}, \ldots, A_{g}\right)$ с координатами

$$
A_{k}=A_{k}\left(\mathbf{z}_{1}, \ldots, \mathbf{z}_{g}\right) \equiv \sum_{j=1}^{g} \Omega_{k}\left(\mathbf{z}_{j}\right) \equiv \sum_{j=1}^{g} \int_{\mathbf{z}_{0}}^{\mathbf{z}_{j}} d \Omega_{k}, \quad k=1, \ldots, g,
$$

также называется отображением Абеля $A: S^{g} \Re \rightarrow$ Јас $\Re$. Если задан вектор $\mathbf{v}=\left(v_{1}, \ldots, v_{g}\right) \in \mathrm{Jac} \mathfrak{R}$, то задача нахождения неупорядоченного набора точек $\left(\mathbf{z}_{1}, \ldots, \mathbf{z}_{g}\right), \mathbf{z}_{j} \in \mathfrak{R}$, для которого

$$
A_{k}\left(\mathbf{z}_{1}, \ldots, \mathbf{z}_{g}\right)=v_{k} \quad(\bmod \text { периодов }), \quad k=1, \ldots, g,
$$

называется проблемой Якоби обрашения абелевых интегралов или просто пробле-

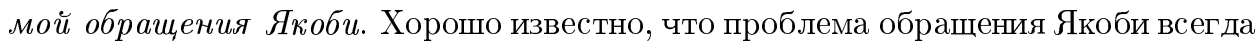
разрешима, но, вообше говоря, не единственным образом.

2. Для любых двух различных точек $\mathbf{z}_{1}$ и $\mathbf{z}_{2}$ римановой поверхности $\mathfrak{R}$ сушествует абелев дифференциал третьего рода, голоморфньй всюду на $\mathfrak{R}$, за исключением этих двух точек, в которых он имеет простые полюсы с вычетами соответственно 1 и -1 ; такой дифференциал называется нормальным. Нормальный дифференциал будет определен однозначно, если потребовать, чтобы все его $a$-периоды были равны нулю; будем называть такой дифференциал $а$-нормированнылм абелевым дифференциалом третьего рода и обозначать $d \Omega\left(\mathbf{z}_{1}, \mathbf{z}_{2} ; \mathbf{z}\right)$; для $a$-нормированного дифференциала $d \Omega\left(\infty^{(1)}, \infty^{(2)} ; \mathbf{z}\right)$ будем иногда для краткости использовать обозначение $d \Omega(\mathbf{z})$ или просто $d \Omega$. Другой способ однозначно задать (нормировать) дифференциал третьего рода - потребовать, чтобы все его периоды были чисто мнимьми. Для таких дифференциалов будем использовать обозначение $d G\left(\mathbf{z}_{1}, \mathbf{z}_{2} ; \mathbf{z}\right)$, предполагая при этом, что вычеты в точках $\mathbf{z}_{1}$ и $\mathbf{z}_{2}$ равны соответственно -1 и 1. К подобньм дифференциалам относится дифференциал комплексной функции Грина $G_{D}(z, \infty)=g_{D}(z, \infty)+i \widetilde{g}_{D}(z, \infty)$ (здесь $\widetilde{g}_{D}(z, \infty)$ - функция, гармонически сопряженная к функции Грина $\left.g_{D}(z, \infty)\right)$, соответствуюшей области $D$. Функция $G_{D}(z, \infty)$ многозначна в $D$ и

$$
G_{D}(z, \infty)=\int_{e_{2 g+2}}^{z} \frac{P_{g}(\zeta)}{\sqrt{h(\zeta)}} d \zeta, \quad z \in D,
$$

где $P_{g}(z)=P_{g}(z ; h)=z^{g}+\cdots \in \mathbb{R}[z]$ - полином, нули которого лежат в лакунах $E_{j}^{\prime}, j=1, \ldots, g$. Дифференциал $d G=d G\left(\infty^{(1)}, \infty^{(2)} ; \mathbf{z}\right)=P_{g}(z) d z / w(\mathbf{z})$ определен на всей римановой поверхности $\mathfrak{R}$, имеет чисто мнимые периоды и простые полюсы в точках $\mathbf{z}=\infty^{(1)}$ и $\mathbf{z}=\infty^{(2)}$ с вычетами соответственно -1 и 1 .

\footnotetext{
${ }^{6} \mathrm{~B}$ дальнейшем мы, как правило, выбираем $\mathbf{z}_{0}=e_{2 g+2}$.
} 
Следовательно, функция $u(\mathbf{z})=\operatorname{Re} \int_{e_{2 g+2}}^{\mathbf{z}} d G$ - однозначная гармоническая функция на $\mathfrak{R} \backslash\left\{\infty^{(1)}, \infty^{(2)}\right\}$, линия уровня которой $\{\mathbf{z}: u(\mathbf{z})=0\}$ совпадает с $L$, а $g_{D}(z, \infty)=u\left(z^{(1)}\right), z \in D$. Как известно, $a$-периоды дифференциала комплексной функции Грина связаны с гармоническими мерами $\omega_{j}(\infty)=\omega\left(E_{j}, \infty ; D\right)$ следующим образом: $\oint_{\mathbf{a}_{k}} d G\left(\infty^{(1)}, \infty^{(2)} ; \mathbf{z}\right)=-2 \pi i \omega_{k}(\infty)$. Кроме того, из вещественности гиперповерхности $\mathfrak{R}$ вытекает, что $\oint_{\mathbf{b}_{k}} d G\left(\infty^{(1)}, \infty^{(2)} ; \mathbf{z}\right)=0$. В дальнейшем нам понадобится следующее соотношение $[4 ;(26)]$ между дифференциалом $d G$ и $a$-нормированным дифференциалом $d \Omega$ :

$$
d G+2 \pi i \sum_{j=1}^{g} \omega_{j}(\infty) d \Omega_{j}+d \Omega=0
$$

Справедливость (8) следует непосредственно из того, что слева в этой формуле стоит голоморфный абелев дифференциал с нулевыми $a$-периодами.

Рассеченная риманова поверхность $\widetilde{\mathfrak{R}}$ получается из $\mathfrak{R}$ проведением сечений по базисным циклам $\mathbf{a}_{k}, \mathbf{b}_{k}, k=1, \ldots, g$, поверхности: $\widetilde{R}=\mathfrak{R} \backslash\left(\bigcup_{k=1}^{g} \mathbf{a}_{k} \cup \bigcup_{k=1}^{g} \mathbf{b}_{k}\right)$; $\widetilde{\Re}$ связна (переход с одного листа на другой осушествляется через $L_{g+1}$ ) и односвязна. Для $b$-периодов $a$-нормированного абелева дифференциала $d \Omega\left(\mathbf{z}_{1}, \mathbf{z}_{2} ; \mathbf{z}\right)$ справедливо следуюшее соотношение Римана [32]:

$$
\int_{\mathbf{b}_{k}} d \Omega\left(\mathbf{z}_{1}, \mathbf{z}_{2} ; \zeta\right)=-2 \pi i \int_{\mathbf{z}_{1}}^{\mathbf{z}_{2}} d \Omega_{k}(\boldsymbol{\zeta})=-2 \pi i\left(\Omega_{k}\left(\mathbf{z}_{2}\right)-\Omega_{k}\left(\mathbf{z}_{1}\right)\right), \quad j=1, \ldots, g,
$$

где интеграл берется по произвольному пути, соединяющему точки $\mathbf{z}_{1}$ и $\mathbf{z}_{2}$ и лежащему в $\widetilde{\Re}$. Используя $(9)$, вычислим $b$-периоды левой части (8). Получим следующее соотношение:

$$
2 \Omega_{k}\left(\infty^{(2)}\right)=\sum_{j=1}^{g} \omega_{j}(\infty) B_{k j}
$$

(напомним, что $\Omega_{k}\left(e_{2 g+2}\right)=0$ в $\left.\widetilde{\Re}\right)$.

3. Дивизором $d$ на римановой поверхности $\Re$ будем называть формальный символ $d=\nu_{1} \mathbf{z}_{1}+\nu_{2} \mathbf{z}_{2}+\cdots+\nu_{m} \mathbf{z}_{m}$, где $\mathbf{z}_{j} \in \mathfrak{R}, \nu_{j} \in \mathbb{Z} ;|d|=\nu_{1}+\cdots+\nu_{m}-$ степень дивизора $d$; если точки $\mathbf{z}_{j}$ попарно различны и все $\nu_{j}>0$, то дивизор $d$ иельй. Произвольной функции $F$, мероморфной на $\mathfrak{R}$ (тем самым, $F \in \mathbb{C}(z, w)$, т.е. $\left.F=r_{1}+r_{2} w, r_{1}, r_{2} \in \mathbb{C}(z)\right)$, сопоставим дивизор $(F)$ ее нулей и полюсов: $(F)=\nu_{1} \mathbf{z}_{1}+\cdots+\nu_{\ell} \mathbf{z}_{\ell}-\mu_{1} \boldsymbol{\zeta}_{1}-\cdots-\mu_{m} \boldsymbol{\zeta}_{m}$, где $\mathbf{z}_{j}, \boldsymbol{\zeta}_{j} \in \mathfrak{R}-$ соответственно нули и полюсы $F$, а $\nu_{j}, \mu_{j} \geqslant 0$ - их кратности; такие дивизоры называются главными. По теореме Абеля дивизор $d=\nu_{1} \mathbf{z}_{1}+\cdots+\nu_{\ell} \mathbf{z}_{\ell}-\mu_{1} \boldsymbol{\zeta}_{1}-\cdots-\mu_{m} \boldsymbol{\zeta}_{m}$ главный тогда и только тогда, когда $|d|=0$ и для всех $k=1, \ldots, g$

$$
\sum_{j=1}^{\ell} \nu_{j} \Omega_{k}\left(\mathbf{z}_{j}\right)-\sum_{j=1}^{m} \mu_{j} \Omega_{k}\left(\boldsymbol{\zeta}_{j}\right)=0 \quad(\bmod \text { периодов })
$$

(здесь пути интегрирования - одни и те же для всех $k$ ). Два дивизора $d_{1}$ и $d_{2}$ эквивалентны, если их разность $d_{1}-d_{2}-$ главный дивизор. Эквивалентные дивизоры 
и только они являются решением проблемы обрашения Якоби для одного и того же вектора из Јас $\mathfrak{R}$. Дивизор $d=\mathbf{z}_{1}+\cdots+\mathbf{z}_{g}$ специальный, если соответствующая проблема обращения Якоби имеет неединственное решение; тем самым, он имеет нетривиальные эквивалентные дивизоры.

Как известно (см., например, [34] и [22]), два целых дивизора степени $g, d_{1}=$ $\mathbf{z}_{1}+\cdots+\mathbf{z}_{g}$ и $d_{2}=\zeta_{1}+\cdots+\boldsymbol{\zeta}_{g}$, заданных на гиперэллиптической римановой поверхности рода $g$, эквивалентны тогда и только тогда, когда $d_{1}=t_{1}^{(1)}+t_{1}^{(2)}+\cdots+$ $t_{2 m}^{(1)}+t_{2 m}^{(2)}+\mathbf{z}_{2 m+1}+\cdots+\mathbf{z}_{g}, d_{2}=\tau_{1}^{(1)}+\tau_{1}^{(2)}+\cdots+\tau_{2 m}^{(1)}+\tau_{2 m}^{(2)}+\mathbf{z}_{2 m+1}+\cdots+\mathbf{z}_{g}$, $m \leqslant g / 2$. Тем самьм, имеется полное описание специальных дивизоров степени $g$. Непосредственно отсюда вытекает следуюшее свойство отображения Абе-

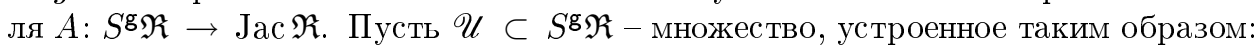
$\mathscr{U}$ представляет собой (неупорядоченное) произведение $g$ непересекающихся компактов $\mathscr{U}_{j}$ в $D^{(1)}, \mathscr{U}_{j} \cap \mathscr{U}_{k}=\varnothing$ при $j \neq k$. Тогда $A^{-1}(A(\mathscr{U}))=\mathscr{U}$ и ограничение $A$ на $\mathscr{U}$ - биекция.

Будем говорить, что дивизор $d^{0}=\mathbf{z}_{1}^{0}+\cdots+\mathbf{z}_{g}^{0} x$-nравильный или просто правильный, если выполняется следующее условие: всякий дивизор с такими же $х$-координатами неспециальный. Иначе говоря, множество

$$
\begin{aligned}
\mathbb{D}=\mathbb{D}\left(x^{0}\right)=\left\{d=\mathbf{z}_{1}\right. & +\cdots+\mathbf{z}_{g}: \sum_{j=1}^{g} \Omega_{k}\left(\mathbf{z}_{j}\right) \\
& \left.\equiv x_{k}^{0}+\sum_{j=1}^{g} \tau_{j} B_{k j}, \tau_{j} \in[0,1), k=1, \ldots, g\right\}
\end{aligned}
$$

состоит из неспециальных дивизоров. Сушествование таких $x$-координат для произвольного $g \in \mathbb{N}$ вытекает из полученного вьше общего вида специальных дивизоров степени $g$ и следующих соображений. Если $d=\mathbf{z}_{1}+\cdots+\mathbf{z}_{g}$ таков, что все $z_{j} \in E_{j}^{\prime}$, то соответствующие $x$-координаты вычисляются по формулам $x_{k}=(g-k+1) / 2(\bmod 1)($ см. [4], [5]). Все такие дивизоры неспециальные и лишь они имеют указанные $x$-координаты. Для случая $g=2$ этот анализ можно существенно уточнить. Действительно, при $g=2$ все специальные дивизоры имеют вид $d=z^{(1)}+z^{(2)}$. Следовательно, $\Omega_{k}\left(z^{(1)}\right)+\Omega_{k}\left(z^{(2)}\right) \equiv 0, k=1$, 2, т.е. для специального дивизора $x$-координаты соответствующего вектора в Јас $\mathfrak{R}$ всегда равны нулю. Тем самым, если при $g=2$ в (11) какая-либо из величин $x_{k}^{0} \neq 0$, то это множество состоит только из неспециальных дивизоров. В таком случае для каждого $d \in \mathbb{D}$ имеем $d=d\left(\tau_{1}, \tau_{2}\right)$, а соответствующие $z_{j}=\psi_{j}\left(\tau_{1}, \tau_{2}\right)$, где $\psi_{j}-$ вещественно аналитические функции переменных $\tau_{1}, \tau_{2}$.

4. Предположим, что на $\mathfrak{R} \backslash L$ задана (кусочно) мероморфная функция $F(\mathbf{z})$, имеюшая полюсы только в бесконечно удаленных точках $\mathbf{z}=\infty^{(1)}$ и $\mathbf{z}=\infty^{(2)}$ каждый порядка $\leqslant g$, предельные значения которой на $L$ сушествуют и удовлетворяют условию:

$$
F^{+}(\boldsymbol{\zeta})-F^{-}(\boldsymbol{\zeta})=\chi(\boldsymbol{\zeta}), \quad \boldsymbol{\zeta} \in L
$$

где функция $\chi$ голоморфна на $L$. Тогда с помощью мероморфного абелева дифференциала

$$
d \Omega(\boldsymbol{\zeta} ; \mathbf{z})=\frac{1}{2} \frac{w(\boldsymbol{\zeta})+w(\mathbf{z})}{\zeta-z} \frac{d \zeta}{w(\boldsymbol{\zeta})}
$$


функция $F(\mathbf{z})$ восстанавливается по граничным условиям (12) интегралом типа Коши:

$$
F(\mathbf{z})=-\frac{1}{2 \pi i} \int_{L^{+}} \chi(\boldsymbol{\zeta}) d \Omega(\mathbf{z} ; \boldsymbol{\zeta})+p(z), \quad \mathbf{z} \notin L,
$$

где $p(z) \in \mathbb{C}[z], \operatorname{deg} p \leqslant g$, а контур $L^{+}$ориентирован положительно относительно области $D^{(1)}$ : область остается слева при обходе по контуру.

Отметим, что при $\boldsymbol{\zeta}=\zeta^{(1)}, \mathbf{z}=z^{(1)}$ или $\boldsymbol{\zeta}=\zeta^{(2)}, \mathbf{z}=z^{(2)}$ для дифференциала (13) имеем

$$
d \Omega\left(z^{(1)} ; \zeta^{(1)}\right)=d \Omega\left(z^{(2)} ; \zeta^{(2)}\right)=\frac{1}{2} \frac{\sqrt{h(\zeta)}+\sqrt{h(z)}}{\zeta-z} \frac{d \zeta}{\sqrt{h(\zeta)}}
$$

а при $\zeta=\zeta^{(2)}, \mathbf{z}=z^{(1)}$

$$
d \Omega\left(z^{(1)} ; \zeta^{(2)}\right)=\frac{1}{2} \frac{\sqrt{h(\zeta)}-\sqrt{h(z)}}{\zeta-z} \frac{d \zeta}{\sqrt{h(\zeta)}}
$$

т.е. в этом случае при $\zeta=z$ дифференциал особенности не имеет.

5. Поясним теперь, что мы понимаем под “начальными условиями, задаваемымм допустимым весом $\rho \neq 0$ и рациональной функиией $r \in \mathbb{C}(z)$ ".

1) Рассмотрим сначала случай, когда $r \equiv 0$, а $\rho=1 / p$, где $p \in \mathbb{C}[z]$. Пусть $p(z)=\prod_{s=1}^{l}\left(z-a_{s}\right), a_{s} \in D$. Пусть $\xi^{0}=\left(\xi_{1}^{0}, \ldots, \xi_{g}^{0}\right)-$ вектор с компонентами

$$
\xi_{k}^{0} \equiv \sum_{s=1}^{l}\left(\Omega_{k}\left(a_{s}^{(2)}\right)-\Omega_{k}\left(\infty^{(2)}\right)\right)-g \Omega_{k}\left(\infty^{(2)}\right)
$$

Соответствуюший дивизор $d^{0}=\mathbf{z}_{1}^{0}+\cdots+\mathbf{z}_{g}^{0}$, удовлетворяющий проблеме обращения Якоби $\sum_{j=1}^{g} \Omega_{k}\left(\mathbf{z}_{j}^{0}\right) \equiv \xi_{k}^{0}, k=1, \ldots, g$, представляет собой начальные условия: $d(0)=d^{0}$. Таким образом, $x$-координаты вектора начальных условий $\xi^{0}$ зависят только от величин $a_{s}$.

2) Пусть теперь $r \equiv 0$, а $\rho \neq 0$ - произвольньй допустимый вес. Определим компоненты $\xi_{k}^{0}$ вектора $\xi^{0}$ соотношением:

$$
\xi_{k}^{0} \equiv \frac{i}{\pi} \int_{E} \log \rho(x) d \Omega_{k}^{+}-g \Omega_{k}\left(\infty^{(2)}\right) .
$$

Дивизор $d^{0}$ определяется как решение проблемы обращения Якоби для вектора $\xi^{0}$. Отметим, что для координат этого вектора в многообразии Якоби имеем:

$$
\begin{gathered}
x_{k}^{0}=-\frac{1}{\pi} \int_{E} \arg \rho(x) d \Omega_{k}^{+}(\bmod 1), \quad y_{k}^{0}=-\frac{g}{2} \omega_{k}(\infty)+\rho_{k}(\bmod 1), \\
\text { где } \sum_{k=1}^{g} \rho_{k} B_{j k}=\frac{i}{\pi} \int_{S} \log |\rho(\zeta)| d \Omega_{j}^{+}(\zeta), \quad j=1, \ldots, g .
\end{gathered}
$$

Эти формулы, разумеется, пригодны и в случае 1). 
3) Рассмотрим, наконец, общий случай $f=\widehat{\rho}+r$. Тогда (см. [4], [5; §§ 3, 4]) необходимо положить

$$
\xi_{k}^{0} \equiv \frac{i}{\pi} \int_{E} \log \rho(x) d \Omega_{k}^{+}-g \Omega_{k}\left(\infty^{(2)}\right)+\sum_{s=1}^{m} \int_{b_{s}^{(2)}}^{b_{s}^{(1)}} d \Omega_{k}, \quad k=1, \ldots, g
$$

где $b_{1}, \ldots, b_{m}-$ полюсы рациональной функции $r$. Так как $\Omega_{k}\left(b_{s}^{(1)}\right)-\Omega_{k}\left(b_{s}^{(2)}\right)=$ $2 \Omega_{k}\left(b_{s}^{(1)}\right)$, то

$$
\xi_{k}^{0}=\frac{i}{\pi} \int_{E} \log \rho(x) d \Omega_{k}^{+}-g \Omega_{k}\left(\infty^{(2)}\right)+2 \sum_{s=1}^{m} \Omega_{k}\left(b_{s}^{(1)}\right) \quad(\bmod \text { периодов }) .
$$

Таким образом, если допустимый вес $\rho$ марковский, то $x$-координаты вектора начальных условий $\xi^{0}$ зависят только от величин $b_{s}$.

\section{§3. Доказательство теорем 1 и 2}

1. Нетрудно видеть, что если полином $p \in \mathbb{C}[z]$ удовлетворяет условиям теоремы 1 , а рациональная дробь $r_{0} \in \mathbb{C}(z)$ равна сумме главных частей функции $1 /(w p)$ в нулях полинома $p$, то мероморфная на $\mathfrak{R}$ функция

$$
f(z)=\frac{1}{w(z) p(z)}-r_{0}(z)
$$

имеет вид $f=\widehat{\rho}$ при $\rho=1 / p$. Функция остатка $R_{n}=R_{n}(z ; \widehat{\rho})$ (см. $\left.\S 1\right)$ также мероморфна на $\mathfrak{R}$, т.е. $R_{n} \in \mathbb{C}(z, w)$. Как известно, таблица Паде произвольной функции состоит из блоков [12], внутри которых рациональные аппроксимации совпадают друг с другом. В соответствии с таким устройством порядок нуля функции остатка $R_{n}$ в бесконечно удаленной точке может быть больше чем $n+1$. Предположим пока, что этот порядок нуля в точности равен $n+1, \operatorname{ardeg} Q_{n}=n$. Kак будет видно в дальнейшем, общий случай вполне укладывается в приводимую ниже схему доказательства.

Итак, функция $R_{n}=R_{n}(\mathbf{z})$ мероморфна на $\Re$ и имеет нуль порядка $n+1$ в бесконечно удаленной точке $\mathbf{z}=\infty^{(1)}$ на первом листе. Тем самьм, мероморфная на $\mathfrak{R}$ функция $w R_{n}$ имеет в бесконечно удаленной точке $\mathbf{z}=\infty^{(1)}$ на первом листе нуль порядка $n-g$ (здесь и всюду в дальнейшем мы считаем, что $n>l=\operatorname{deg} p, n>g)$. Из представления (16) для функции $f=\widehat{\rho}$ получаем следуюшее соотношение для значений этой функции в сопряженных точках $z^{(1)}$ и $z^{(2)}$ на $\Re$ :

$$
f\left(z^{(2)}\right)=\frac{1}{w\left(z^{(2)}\right) p(z)}-r_{0}(z)=-\frac{1}{w\left(z^{(1)}\right) p(z)}-r_{0}(z)=f\left(z^{(1)}\right)-\frac{2}{w\left(z^{(1)}\right) p(z)}
$$

откуда вытекает, что

$$
R_{n}\left(z^{(2)}\right)=R_{n}\left(z^{(1)}\right)-\frac{2}{w\left(z^{(1)}\right) p(z)} Q_{n}(z)
$$

и

$$
\left(w R_{n}\right)\left(z^{(2)}\right)=-\left(w R_{n}\right)\left(z^{(1)}\right)+\frac{2}{p(z)} Q_{n}(z), \quad z \in D
$$


Функция $f$, а тем самым и $R_{n}$ голоморфны в $D$. Следовательно, функция $F_{n}(\mathbf{z})=$ $\left(w R_{n}\right)(\mathbf{z})$ голоморфна на первом листе $D^{(1)}$, где она имеет нуль порядка $n-g$ в точке $\mathbf{z}=\infty^{(1)}$, и на кривой $L$. Из $(17)$ вытекает, что $F_{n}$ имеет полюсы на втором листе $D^{(2)}$ в точках $a_{j}^{(2)}, j=1, \ldots, l$, соответствующих нулям полинома $p$ (здесь мы предполагаем, также без ограничения общности, что $\left.Q_{n}\left(a_{j}\right) \neq 0\right)$, и полюс кратности $n-l$ в точке $\infty^{(2)}$. По теореме Абеля число нулей произвольной функции класса $\mathbb{C}(z, w)$ в точности равно числу ее полюсов. Следовательно, функция $F_{n}$ имеет еше ровно $g$ свободных нулей на $\mathfrak{R}$ и для дивизора $\left(F_{n}\right)$ справедлива формула:

$$
\left(F_{n}\right)=(n-g) \infty^{(1)}+\mathbf{z}_{1}+\cdots+\mathbf{z}_{g}-(n-l) \infty^{(2)}-a_{1}^{(2)}-\cdots-a_{l}^{(2)},
$$

где $\mathbf{z}_{1}, \ldots, \mathbf{z}_{g}$ - некоторые точки на $\mathfrak{R}$, которые, разумеется, зависят от $n$. Нетрудно видеть, что полученная формула охватывает и общий случай. Действительно, тот факт, что функция остатка имеет дополнительньй нуль в бесконечно удаленной точке, соответствует тому, что $\mathbf{z}_{j}=\infty^{(1)}$ при некотором $j \in\{1, \ldots, g\}$. Аналогично трактуется и ситуация, когда $\operatorname{deg} Q_{n}<n$ или $Q_{n}\left(a_{j}\right)=0$. Заметим наконец, что если обе точки вида $\zeta^{(1)}$ и $\zeta^{(2)}$ являются нулями функции $F_{n}$, то из $(17)$ немедленно вытекает, что $R_{n}$ и $Q_{n}$ имеют общий нуль $z=\zeta$, чего не может быть в случае нормального индекса $n$.

Из теоремы Абеля вытекает, что для точек $\mathbf{z}_{1}, \ldots, \mathbf{z}_{g}$ должны выполняться следуюшие соотношения (cp. $\S 2$, п. 3):

$(n-g) \Omega_{k}\left(\infty^{(1)}\right)+\sum_{j=1}^{g} \Omega_{k}\left(\mathbf{z}_{j}\right)-(n-l) \Omega_{k}\left(\infty^{(2)}\right)-\sum_{s=1}^{l} \Omega_{k}\left(a_{s}^{(2)}\right) \equiv 0, k=1, \ldots, g$

где пути интегрирования - одни и те же для всех $k$. Непосредственно отсюда получаем систему из $g$ уравнений на $g$ точек $\mathbf{z}_{1}, \ldots, \mathbf{z}_{g}$

$$
\sum_{j=1}^{g} \Omega_{k}\left(\mathbf{z}_{j}\right) \equiv 2 n \Omega_{k}\left(\infty^{(2)}\right)+\sum_{s=1}^{l}\left(\Omega_{k}\left(a_{s}^{(2)}\right)-\Omega_{k}\left(\infty^{(2)}\right)\right)-g \Omega_{k}\left(\infty^{(2)}\right), k=1, \ldots, g
$$

К этой системе мы вернемся чуть позже, а сейчас получим явное представление для функции $F_{n}$ в терминах абелевых дифференциалов. Рассмотрим следующий дифференциал третьего рода на $\mathfrak{R}$ с чисто мнимьми периодами:

$$
d U_{n}(\mathbf{z})=n d G\left(\infty^{(1)}, \infty^{(2)} ; \mathbf{z}\right)+\sum_{j=1}^{g} d G\left(\mathbf{z}_{j}, \infty^{(1)} ; \mathbf{z}\right)+\sum_{s=1}^{l} d G\left(\infty^{(2)}, a_{j}^{(2)} ; \mathbf{z}\right) .
$$

Нетрудно видеть, что абелев дифференциал $d \log F_{n}+d U_{n}$ голоморфен на $\mathfrak{R}$, а все его периоды чисто мнимые. Как хорошо известно, такой дифференциал тождественно равен нулю. Итак, получаем следующее представление для функции $F_{n}$ (cp. [20]):

$$
F_{n}(\mathbf{z})=\Phi^{-n}(\mathbf{z}) \exp \left\{-\sum_{j=1}^{g} G\left(\mathbf{z}_{j}, \infty^{(1)} ; \mathbf{z}\right)-\sum_{s=1}^{l} G\left(\infty^{(2)}, a_{j}^{(2)} ; \mathbf{z}\right)\right\}
$$


где $\Phi(\mathbf{z})=\exp \{G(\mathbf{z})\}$ - так называемая (многозначная) отображающая функция: $\left|\Phi^{ \pm}(x)\right|=1$ при $x \in E,|\Phi(z)|>1$ при $z \in D$. Поясним: предполагается, что при переходе от дифференциала $d \log F_{n}+d U_{n}$ к функции $\log F_{n}+U_{n}$ интегрирование происходит по произвольному пути, соединяющему точки $e_{2 g}+2$ и $\mathbf{z}$, не проходящему через особенности этих дифференциалов и целиком лежащему в рассеченной римановой поверхности $\widetilde{\Re}$. Тем самым, многозначная функция $U_{n}$ нормирована условием $U_{n}\left(e_{2 g+2}\right)=0(\bmod 2 \pi i)$, а функция $F_{n}=e^{-U_{n}}-$ условием $F_{n}\left(e_{2 g+2}\right)=1$. Таким образом, $\left(w R_{n}\right)\left(e_{2 g+2}\right)=1$ и

$$
\left(w R_{n}\right)\left(z^{(1)}\right)=\Phi^{-n}\left(z^{(1)}\right) \exp \left\{-\sum_{j=1}^{g} G\left(\mathbf{z}_{j}, \infty^{(1)} ; z^{(1)}\right)-\sum_{s=1}^{l} G\left(\infty^{(2)}, a_{j}^{(2)} ; z^{(1)}\right)\right\}
$$

Определим теперь для $z \in D$ функцию $V_{n}(z):=F_{n}\left(z^{(1)}\right) F_{n}\left(z^{(2)}\right)$. Нетрудно видеть, что для ее дивизора в $D$ имеем: $\left(V_{n}\right)_{D}=(l-g) \infty+z_{1}+\cdots+z_{g}-a_{1}-\cdots-a_{l}$, а сама функция $V_{n}$ продолжается как мероморфная (т.е. однозначная аналитическая) функция в $\overline{\mathbb{C}} \backslash\left\{e_{1}, \ldots, e_{2 g+2}\right\}$. Отсюда вытекает, что $V_{n}$ однозначна в $\overline{\mathbb{C}}_{и}$ $V_{n}(z)=X_{n}(z) / p_{l}(z)$, где нормировка полинома $X_{n}=\mathrm{const} \prod_{j=1}^{g}\left(z-z_{j}\right)$ согласована с ранее выбранной нормировкой $V_{n}\left(e_{2 g+2}\right)=1$.

Из $(17)$, умножив предварительно обе его части на $\left(w R_{n}\right)\left(z^{(1)}\right)$ и учитьвая только что установленные свойства функции $V_{n}$, а также соглашение об отождествлении $z$ и $z^{(1)}$ для $z \in D$, получаем следующее представление:

$$
Q_{n}(z)\left(w R_{n}\right)(z)=\frac{1}{2}\left\{p_{l}(z)\left(w R_{n}\right)^{2}(z)+X_{n}(z)\right\}, \quad z \in D .
$$

Тем самым фактически получена явная формула для полиномов $Q_{n}$ в терминах функции остатка $R_{n}$, мероморфной на $\mathfrak{R}$, и полиномиального параметра $X_{n}$.

Из представления (19) для функции $F_{n}$ вытекает, что первое слагаемое в правой части (20) убывает с геометрической скоростью по сравнению со вторьм. Тем самьм, по теореме Руше имеем: на любом компакте число нулей (голоморфной) функции $Q_{n} w R_{n}$ и функции $X_{n} / 2$ одинаково. Нули функции остатка $R_{n}$ в $D-$ это те и только те нули $X_{n}$, которые порождены точками $z_{j}^{(1)}$. На основании полученного ранее представления $\left(w R_{n}\right)\left(z^{(1)}\right) \cdot\left(w R_{n}\right)\left(z^{(2)}\right)=X_{n}(z) / p_{l}(z)$ мы можем считать нулями функции остатка и те нули $X_{n}$, которые попали на компакт $E$. Оставшиеся нули $X_{n}$ или стремятся к $E$, или компактно по $n$ принадлежат $D$ и произошли от точек $z_{j}^{(2)}$ (т.е. это точки $\operatorname{pr} z_{j}^{(2)}$ ). Очевидно, нули $X_{n}$ второй группы и только они соответствуют тем нулям полинома $Q_{n}$, которые компактно принадлежат $D$. Тем самым, нули полинома $Q_{n}$, имеющие предельные точки вне множества E, полностью описаны в терминах соответствующих нулей полинома $X_{n}$, где, напомним, нули полинома $X_{n}-$ в точности проекции $z_{j}$ точек $\mathbf{z}_{j}=\left(z_{j}, \pm\right) \in \mathfrak{R}$, составляющих дивизор $d$. Отсюда, в частности, вытекает, что число таких нулей знаменателя $\leqslant g$. Отметим, что при доказательстве результатов этого пункта условие правильности дивизора $d^{0}$ нигде не использовалось.

2. Вернемся к системе (18) уравнений на дивизор $d$. Запишем ее в следуюшем виде:

$$
\sum_{j=1}^{g} \Omega_{k}\left(\mathbf{z}_{j}\right)=\xi_{k} \quad(\bmod \text { периодов }), \quad k=1, \ldots, g,
$$


где

$$
\xi_{k}=2 n \Omega_{k}\left(\infty^{(2)}\right)+\sum_{s=1}^{l}\left(\Omega_{k}\left(a_{s}^{(2)}\right)-\Omega_{k}\left(\infty^{(2)}\right)\right)-g \Omega_{k}\left(\infty^{(2)}\right)
$$

Соотношения (21) представляют собой проблему обрашения Якоби для вектора $\xi=\left(\xi_{1}, \ldots, \xi_{g}\right)$.

Рассмотрим проблему обращения Якоби для вектора $\xi^{0}=\left(\xi_{1}^{0}, \ldots, \xi_{g}^{0}\right)$, где координаты $\xi_{k}^{0}$ заданы соотношениями (ср. с (22))

$$
\xi_{k}^{0}=\sum_{s=1}^{l}\left(\Omega_{k}\left(a_{s}^{(2)}\right)-\Omega_{k}\left(\infty^{(2)}\right)\right)-g \Omega_{k}\left(\infty^{(2)}\right)
$$

Решение $d^{0}=\mathbf{z}_{1}^{0}+\cdots+\mathbf{z}_{g}^{0}$ такой проблемы всегда сушествует, а если дивизор $d^{0}$ неспециальный, то оно единственно. По условию теоремы дивизор $d^{0}$ неспециальный. Пусть координаты вектора $\xi^{0}$ в многообразии Якоби равны $x_{j}^{0}, y_{j}^{0}$. Тем самьм, для дивизора $d^{0}=\mathbf{z}_{1}^{0}+\cdots+\mathbf{z}_{g}^{0}$ имеем

$$
\sum_{j=1}^{g} \Omega_{k}\left(\mathbf{z}_{j}^{0}\right)=x_{k}^{0}+\sum_{j=1}^{g} y_{j}^{0} B_{k j} \quad(\bmod \text { периодов }), \quad k=1, \ldots, g .
$$

Пользуясь этим соотношением и формулой (10), приведем представления (22) для $\xi_{k}$ в уравнениях $(21)$ к виду

$$
\xi_{k}=x_{k}^{0}+\sum_{j=1}^{g} y_{j}^{0} B_{k j}+n \sum_{j=1}^{g} \omega_{j}(\infty) B_{k j}, \quad k=1, \ldots, g
$$

т.е. $x$-, $y$-координаты вектора $\xi=\left(\xi_{1}, \ldots, \xi_{g}\right)$ в многообразии Якоби вычисляются по формулам

$$
x_{j}=x_{j}^{0}, \quad y_{j}=y_{j}^{0}+n \omega_{j}(\infty) \quad(\bmod 1), \quad j=1, \ldots, g
$$

Рассмотрим в Јас $\mathfrak{R}$ множество точек с координатами

$$
x_{k}=x_{k}^{0}, \quad y_{k}=\tau_{k}, \quad k=1, \ldots, g, \quad \text { где } \tau_{k} \in[0,1)
$$

изоморфное $g$-мерному вешественному тору $\mathbb{T}^{g}$. Пусть $\mathbb{D}=\mathbb{D}\left(x^{0}\right)$ - соответствующее ему множество в $S^{g} \mathfrak{R}$ :

$$
\mathbb{D}=\left\{d=\mathbf{z}_{1}+\cdots+\mathbf{z}_{g}: \sum_{j=1}^{g} \Omega_{k}\left(\mathbf{z}_{j}\right) \equiv x_{k}^{0}+\sum_{j=1}^{g} \tau_{j} B_{k j}, \tau_{j} \in[0,1), k=1, \ldots, g\right\}
$$

По условию координаты $x_{k}^{0}$ дивизора $d^{0}$ таковы, что всякий дивизор $d$ из $\mathbb{D}$ неспециальный. Тем самым, каждый дивизор $d \in \mathbb{D}$ однозначно определяется заданньми $\tau_{k} \in[0,1): d=d(\tau)=d\left(\tau_{1}, \ldots, \tau_{g}\right)$, т.е. $\mathbb{D}$ также изоморфно $\mathbb{T}^{g}$.

$\mathrm{B}$ дальнейшем нам понадобятся некоторые свойства множества $\mathbb{D}$. 
ЛЕмма 1. Замкнутое множсество $\mathbb{D} \subset S^{g} \mathfrak{R}$ обладает следующими свойствами:

1) если дивизор $\mathbf{z}_{1}+\cdots+z_{j}^{(1)}+\cdots+\mathbf{z}_{g}=d(\tau) \in \mathbb{D}$ при некотором $\tau=$ $\left(\tau_{1}, \ldots, \tau_{g}\right)$, то дивизор $\mathbf{z}_{1}+\cdots+\bar{z}_{j}^{(2)}+\cdots+\mathbf{z}_{g}=d\left(\tau^{\prime}\right) \in \mathbb{D}$ при некотором $\tau^{\prime}=\left(\tau_{1}^{\prime}, \ldots, \tau_{g}^{\prime}\right)$

2) функции $\mathbf{z}_{j}=\psi_{j}\left(\tau_{1}, \ldots, \tau_{g}\right)$ - вещественно аналитические функции переменных $\tau_{j}$, удовлетворяющие системе дифференииальных уравнений

$$
\left(d \psi_{1}, \ldots, d \psi_{g}\right)^{T}=\left(\Omega_{k}^{\prime}\left(\psi_{j}\right)\right)^{-1} \mathbf{B}\left(d \tau_{1}, \ldots, d \tau_{g}\right)^{T} .
$$

Отметим, что это соотношение на функции $\psi_{k}$ переменных $\tau_{j}$, записанное в формe

$$
\left\|\Omega_{k}^{\prime}\left(\psi_{j}\right)\right\|\left\|\frac{\partial \psi_{k}}{\partial \tau_{j}}\right\|=\mathbf{B},
$$

задает естественное обобшение понятия ортогональной траектории квадратичного дифференциала на римановой поверхности (см. [35]). Действительно, ортогональная траектория квадратичного дифференциала $Q(\zeta) d \zeta^{2}-$ (максимальная) кривая, на которой $Q(\zeta) d \zeta^{2}<0$, т.е. $\sqrt{Q(\zeta)} d \zeta \in i \mathbb{R}$ или, при подходящей параметризации, $\sqrt{Q(\zeta)} d \zeta / d \tau=i$. Естественность обобшения вытекает теперь из того, что матрица В чисто мнимая, при этом $\operatorname{Im} \mathbf{B}>0$.

ДокАЗАТЕЛЬСТво ЛЕммы 1. Утверждения п. 2) следуют непосредственно из определения множества $\mathbb{D}$.

Для доказательства п. 1) рассмотрим квадратичный дифференциал $d \Omega_{k}^{2}, k=$ $1, \ldots, g$, порожденный голоморфным абелевым дифференциалом $d \Omega_{k}$, и воспользуемся хорошо известными [35; гл. III] свойствами квадратичных дифференциалов на римановой поверхности. Из теоремы о локальном строении их траекторий вытекает, что через каждую точку $\mathfrak{R}$, которая не является критической для квадратичного дифференциала, проходит ровно одна ортогональная траектория. Критические точки $d \Omega_{k}^{2}$ - это в точности его нули, которые все двукратны и лежат на $g-1$ отрез$\operatorname{ках~} E_{j}, j \neq k$. Тем самым, через любую точку $z^{(1)} \in D^{(1)}$ проходит ровно по одной ортогональной траектории $\ell_{k}$ для каждого дифференциала $d \Omega_{k}^{2}$. Из теоремы о глобальном строении траекторий [35; гл. III, $\S 3.4$, теорема 3.5$]$ вытекает, что каждая из кривых $\ell_{k}$ проходит и через точку $\bar{z}^{(2)}$. Тем самым, $\Omega_{k}\left(z^{(1)}\right)-\Omega_{k}\left(\bar{z}^{(2)}\right) \in i \mathbb{R}$ $(\bmod$ периодов) для любого $k=1, \ldots, g$. Отсюда, так как матрица $\mathbf{B}$ невырождена, вытекает справедливость 1).

3. Пусть

$$
\begin{aligned}
& \mathbb{D}_{1}=\left\{d(\tau) \in \mathbb{D}: \tau_{j}=y^{0}+t \omega_{j}(\infty)(\bmod 1), t \in \mathbb{R}_{+}, j=1, \ldots, g\right\} \\
& \mathbb{D}_{0}=\left\{d(\tau) \in \mathbb{D}: \tau_{j}=y^{0}+n \omega_{j}(\infty)(\bmod 1), n \in \mathbb{N}, j=1, \ldots, g\right\}
\end{aligned}
$$

$\mathbb{D}_{0} \subset \mathbb{D}_{1} \subset \mathbb{D} ; \mathbb{D}_{1}=\left\{d(t)=\mathbf{z}_{1}(t)+\cdots+\mathbf{z}_{g}(t)\right\}$ - кривая в $\mathbb{D}, \mathbb{D}_{0}=\left\{d(n)=\mathbf{z}_{1}(n)+\right.$ $\left.\cdots+\mathbf{z}_{g}(n)\right\}$ - дискретное множество. Движение дивизора $d(t)=\mathbf{z}_{1}(t)+\cdots+\mathbf{z}_{g}(t)$ на торе $\mathbb{D}$ - условно периодическое с периодами $\omega_{1}(\infty), \ldots, \omega_{g}(\infty)($ см., например, $[36 ;$ гл. $10, \S 51])$.

$\mathrm{C}$ точки зрения изучения сходимости аппроксимаций Паде нас интересует множество предельных точек $\mathbb{D}_{0}^{\prime}$. Отметим следующий хорошо известный факт $[36$; гл. $10, \S 51]$ : если величины $\omega_{1}(\infty), \ldots, \omega_{g}(\infty)$ рационально независимы, то $\mathbb{D}_{1}^{\prime}=\mathbb{D}$, 
т.е. траектория $\mathbb{D}_{1}$ дивизора $d(t)$ всюду плотна в $\mathbb{D}$; если же $\omega_{1}(\infty), \ldots, \omega_{g}(\infty), 1$ рационально независимы ${ }^{7}$, то и $\mathbb{D}_{0}^{\prime}=\mathbb{D}$.

Из леммы 1 следует, что функции $z_{j}=z_{j}(t)$ - вешественно аналитические функции переменной $t \in \mathbb{R}$, поэтому для $d \in \mathbb{D}_{1}$ получаем:

$$
\sum_{j=1}^{g} \Omega_{k}^{\prime}\left(\mathbf{z}_{j}\right) \dot{z}_{j}=\sum_{j=1}^{g} \omega_{j}(\infty) B_{j k}, \quad k=1, \ldots, g,
$$

где $\dot{z}_{k}=d z_{k} / d t$. Рассмотрим (23) как линейную систему относительно $\dot{z}_{j}$. Нетрудно видеть, что

$$
\operatorname{det}\left\|\Omega_{k}^{\prime}\left(\mathbf{z}_{j}\right)\right\|=\frac{\text { const }}{\prod_{j=1}^{g} w\left(\mathbf{z}_{j}\right)} V\left(z_{1}, \ldots, z_{g}\right)
$$

где const зависит только от точек $e_{1}, \ldots, e_{2 g+2}, V$ - определитель Вандермонда от $g$ переменных. Пусть $D_{k}$ - определитель матрицы, полученной из матрицы системы (23) заменой $k$-го столбца на столбец правых частей. С помощью соотношения (10) получаем:

$$
D_{k}=-\frac{\mathrm{const}}{2} \int_{e_{2 g+2}}^{+\infty} \frac{V_{k}\left(z_{1}, \ldots, x, \ldots, z_{g}\right)}{\prod_{j \neq k} w\left(\mathbf{z}_{j}\right)} \frac{d x}{w(x)}
$$

где const - та же, что и в (24), путь интегрирования - луч от $e_{2 g+2}$ до $+\infty, V_{k}$-определитель Вандермонда от $g$ переменных, в котором $z_{k}$ заменено на $x$. Разрешая теперь систему (23) относительно величин $\dot{z}_{j}$ и используя только что установленные формулы (24), (25), а также явную формулу для определителя Вандермонда, получаем динамическую систему (*). Система Дубровина [37] (см. также [38; гл. VIII, $(8.2 .15)])$ получается из $(*)$ заменой сомножителя $\left(z-e_{2 g+2}\right)$ на $\left(z / e_{2 g+2}-1\right)$ и последуюшим (формальным) переходом к пределу при $e_{2 g+2} \rightarrow \infty$.

4. Положим $\vec{\Omega}(z)=\left(\Omega_{1}(z), \ldots, \Omega_{g}(z)\right)^{T}, \vec{\omega}(z)=\left(\omega_{1}(z), \ldots, \omega_{g}(z)\right)^{T}$, где $z \in D, \omega_{j}(z)=\omega\left(E_{j}, z ; D\right)$. Нетрудно проверить, что справедливо следующее представление:

$$
\operatorname{Re}\left\{\mathbf{B}^{-1} \vec{\Omega}(z)\right\}=-\frac{1}{2} \vec{\omega}(z), \quad z \in D
$$

С учетом этой формулы и $(10)$ для дивизора $d(t)=\mathbf{z}_{1}(t)+\cdots+\mathbf{z}_{g}(t) \in \mathbb{D}_{1}$ получаем следуюшее соотношение:

$$
\sum_{j=1}^{g} \varepsilon_{j} \omega_{s}\left(z_{j}\right)=(g-2 t) \omega_{s}(\infty)+2 \rho_{s} \quad(\bmod 2), \quad s=1, \ldots, g,
$$

где $\varepsilon_{j}= \pm 1$ в зависимости от положения точки $\mathbf{z}_{j}=\left(z_{j}, \pm\right)$ на $\mathfrak{R} . \mathrm{B}$ частности, если при переходе от $t=n \mathrm{k} t=n+1$ все знаки всех величин $\varepsilon_{j}$ сохраняются, то

$$
\sum_{j=1}^{g} \varepsilon_{j} \Delta \omega_{s}\left(z_{j}\right)=-2 \omega_{s}(\infty)
$$

\footnotetext{
${ }^{7}$ В силу соотношения $\omega_{1}(\infty)+\cdots+\omega_{g+1}(\infty)=1$ это условие эквивалентно тому, что $\omega_{1}(\infty), \ldots, \omega_{g+1}(\infty)$ рационально независимы.
} 
(ср. [7; лемма 3] и [39; с. 62]). Соотношения (27) фактически означают, что движение дивизора $d(n)=\mathbf{z}_{1}(n)+\cdots+\mathbf{z}_{g}(n)$ по кривой $\mathbb{D}_{1}$ происходит с постоянной "угловой скоростью".

Отметим следующий любопытный факт, связанный с гармоническими мерами $\omega_{j}$. Так как область $D$ многосвязна, то гармонически сопряженные функции $\widetilde{\omega}_{j}$ многозначны. Соответствующие величины $P_{j k}=\vec{E}_{k} \widetilde{\omega}_{j}-$ периоды функции $\widetilde{\omega}_{j}$ относительно $E_{k}$ - впервые рассматривались Максвеллом [40; часть I, гл. III, пп. 87-89] (см. также [41]), который, в частности, доказал, что матрица $\mathbf{P}=\left\|P_{j k}\right\|$ симметрична и положительно определена. Непосредственно из (26) вытекает, что в случае вешественной римановой поверхности матрицы $\mathbf{B}$ и $\mathbf{P}$ связаны соотношением: $\frac{1}{2} \operatorname{Im} \mathbf{B} \cdot \mathbf{P}=\mathbf{1}$.

5. Подведем итоги. В условиях теоремы 1 при каждом $n \in \mathbb{N}$ нули полинома $X_{n}$ - это в точности проекции на $\mathbb{C}$ точек $\mathbf{z}_{j}(n)$ для $n \in \mathbb{N}$ и $j=1, \ldots, g$. При произвольном $t \in \mathbb{R}_{+}$точки $\mathbf{z}_{j}(t)$ составляют дивизор $d(t)=\mathbf{z}_{1}(t)+\cdots+\mathbf{z}_{g}(t) \in \mathbb{D}_{1}$, $x$-координаты которого постоянны и полностью определяются начальньми условиями задачи (т.е. полиномом $p \in \mathbb{C}[z])$, а $y$-координаты равны $y_{j}=y_{j}^{0}+t \omega_{j}(\infty)$ $(\bmod 1)$ (и, тем самым, есть условно периодические функции переменной $t)$. Дивизор $d(t)$ неспециален и удовлетворяет системе $(*)$.

Непосредственно из пп. 1-4 настояшего параграффа вытекает справедливость теорем 1 и 2.

6. Обозначим через $\mathscr{P}$ множество предельных точек полюсов аппроксимаций Паде, принадлежащих $D$, а через $\mathscr{Z} \subset D$-множество предельных точек свободных нулей функции остатка $R_{n}$. Пусть $\mathfrak{D} \subset \mathfrak{R}-$ полньй прообраз множества $\mathbb{D}\left(x^{0}\right)$ при отображении $\mathfrak{R} \rightarrow S^{g} \mathfrak{R}, \mathfrak{D}^{(1)}$ и $\mathfrak{D}^{(2)}$ - его части, расположенные соответственно на первом и втором (открытых) листах $\mathfrak{R}$. Из полученных результатов вытекает, что в условиях теоремы 1 множество $\mathscr{P} \subset \operatorname{pr} \mathfrak{D}^{(2)}$, а $\mathscr{Z} \subset \operatorname{pr} \mathfrak{D}^{(1)}$. Если, кроме того, выполнено условие рациональной независимости величин $\omega_{1}(\infty), \ldots, \omega_{g+1}(\infty)$, то $\mathscr{P}=\operatorname{pr} \mathfrak{D}^{(2)}$, а $\mathscr{Z}=\operatorname{pr} \mathfrak{D}^{(1)}$. Более того, при этом условии всякий дивизор $d=$ $z_{1}^{(2)}+\cdots+z_{g}^{(2)} \in \mathbb{D}-$ предельная точка для дивизоров, составленных из полюсов аппроксимаций Паде, а всякий дивизор $d=z_{1}^{(1)}+\cdots+z_{g}^{(1)} \in \mathbb{D}$ - предельная точка для дивизоров, составленных из свободных нулей $R_{n}$.

СЛЕДСТВИЕ 1. Если при всех $n \in \Lambda \subset \mathbb{N}$ в дивизоре $d(n)=\mathbf{z}_{1}(n)+\cdots+$ $\mathbf{z}_{g}(n)$ ровно $k \leqslant g$ точек $\mathbf{z}_{j}(n)$ компактно по $n$ принадлежсат $D^{(2)}$, то по этой подпоследовательности $\Lambda$ нули $Q_{n}$ имеют в точности $k$ предельных точек вне E. Аналогичное утвержсение с заменой $D^{(2)}$ на $D^{(1)}$ справедливо для $R_{n}$.

Для произвольного дивизора $d=\mathbf{z}_{1}+\cdots+\mathbf{z}_{g}$ под $\operatorname{pr} d$ будем понимать дивизор $z_{1}+\cdots+z_{g}$

СЛЕДСТВИЕ 2. Если величины $\omega_{1}(\infty), \ldots, \omega_{g+1}(\infty)$ рачионально независимьи, то любой дивизор $\operatorname{pr} d$, где $d \in \mathbb{D}$, есть предельная точка последовательности дивизоров степени $g$, составленных из нулей произведения $Q_{n} R_{n}$, располохсенньх в $\mathbb{C} \backslash E$.

СлЕДСТВИЕ 3. Если дивизор $d^{0}$ таков, что все $z_{j}^{0} \in E_{j}^{\prime}, j=1, \ldots, g$, то $\mathbb{D}\left(x^{0}\right)=\ell_{1} \times \cdots \times \ell_{g}$, әде $\ell_{j}-$ замкнутые кривые на $\mathfrak{R}$, соответствующие лакунам $E_{j}^{\prime}$. 
Утверждение следствия 3 вытекает непосредственно из следующего легко проверяемого факта: дивизор $d^{0}$ имеет $x$-координаты, равные $x_{k}^{0}=(g-k+1) / 2$ $(\bmod 1)$ тогда и только тогда, когда все его точки лежат в лакунах: $z_{j}^{0} \in E_{j}^{\prime}($ см. $[5 ; \oint 4])$.

Отметим, что соответствующие отрезкам $E_{j}$ замкнутые кривые $L_{j} \subset \Re$ траектории квадратичных дифференциалов $d \Omega_{k}^{2}$, а $\ell_{j}$ - ортогональные траектории $d \Omega_{k}^{2}$.

\section{§4. Доказательство теоремы 3}

1. Доказательство теоремы 3 проведем по схеме, впервые реализованной в [4] (см. также [5]), в основе которой - “явное" построение и изучение свойств решения определенной краевой задачи Римана на $\mathfrak{R}$.

Пусть $\Lambda=\Lambda_{1}$ или $\Lambda=\Lambda_{2}$ в соответствии с п. $1^{\circ}$ или п. $2^{\circ}$. Рассмотрим сначала случай, когда $r \equiv 0, \rho \neq 0$ - допустимый вес. Стандартным образом [4], [5] доказывается, что для любого $n \in \Lambda$ сушествует кусочно мероморфная на $\mathfrak{R}$ функция $\Psi_{n} \in \mathscr{M}(\mathfrak{R} \backslash L)$ такая, что:

1) дивизор $\left(\Psi_{n}\right)=-n \infty^{(2)}+\mathbf{z}_{1}+\cdots+\mathbf{z}_{g}+(n-g) \infty^{(1)}$, где $\mathbf{z}_{j}=\mathbf{z}_{j}(n) \in \Re \backslash L$, и, кроме того, выполняется краевое условие

2) $\Psi^{+}(\boldsymbol{\zeta})=\rho(\zeta) \Psi^{-}(\boldsymbol{\zeta}), \boldsymbol{\zeta} \in L$.

Для $z \in D$ положим: $\varphi_{n}(z):=\Psi_{n}\left(z^{(2)}\right), \psi_{n}(z):=\Psi_{n}\left(z^{(1)}\right)$. Получим пару функций $\left(\varphi_{n}, \psi_{n}\right)$, которая играет ключевую роль в рассуждениях работ [4], [5]. Повторим коротко схему доказательства работы [4]. Функции $\varphi_{n}, \psi_{n}$ мероморфны в $D$ и удовлетворяют краевым условиям на $E: \rho \varphi_{n}^{+}=\psi_{n}^{-}, \rho \varphi_{n}^{-}=\psi_{n}^{+}$. Для их дивизоров в $D$ имеем: $\left(\varphi_{n}\right)=-n \infty+z_{1}+\cdots+z_{\varkappa},\left(\psi_{n}\right)=(n-g) \infty+z_{\varkappa+1}+\cdots+z_{g}$, где $\varkappa=\varkappa(n), 0 \leqslant \varkappa \leqslant g$, причем $\varkappa \leqslant g-1$ при $\Lambda=\Lambda_{1}, \varkappa \geqslant 1$ при $\Lambda=\Lambda_{2}$.

Непосредственно из формулы Сохоцкого для предельных значений интеграла типа Коши вытекает следуюшее соотношение для функции остатка $R_{n}$ на множестве $E$ :

$$
R_{n}^{+}(\zeta)-R_{n}^{-}(\zeta)=-\frac{Q_{n}(\zeta) \rho(\zeta)}{w^{+}(\zeta)}, \quad \zeta \in E \backslash\left\{e_{1}, \ldots, e_{2 g+2}\right\}
$$

Из этого соотношения, используя условия на функции $\varphi_{n}$ и $\psi_{n}$, легко получаем следующую пару соотношений:

$$
\begin{array}{ll}
\left(R_{n} \varphi_{n} w\right)^{+}(\zeta)+\left(R_{n} \psi_{n} w\right)^{-}(\zeta) \frac{1}{\rho(\zeta)}=-Q_{n}(\zeta) \psi_{n}^{-}(\zeta), & \zeta \in E \\
\left(R_{n} \varphi_{n} w\right)^{-}(\zeta)+\left(R_{n} \psi_{n} w\right)^{+}(\zeta) \frac{1}{\rho(\zeta)}=-Q_{n}(\zeta) \psi_{n}^{+}(\zeta), \quad \zeta \in E
\end{array}
$$

Определим теперь на двулистной римановой поверхности $\Re$ кусочно мероморфиную функцию $F(\mathbf{z})$ следующим образом:

$$
F(\mathbf{z})= \begin{cases}R_{n}(z) \varphi_{n}(z) w(z), & \mathbf{z} \in D^{(1)} \\ -Q_{n}(z) \psi_{n}(z), & \mathbf{z} \in D^{(2)}\end{cases}
$$

Нетрудно увидеть, что в бесконечно удаленных точках $\mathbf{z}=\infty^{(1)}$ и $\mathbf{z}=\infty^{(2)}$ функция $F$ имеет полюсы порядка $\leqslant g$; в остальных точках $\Re \backslash L$ функция $F$ голоморфна. 
Непосредственно из (28) вытекает, что на кривой $L$ для $F$ выполняется граничное условие

$$
F^{+}(\boldsymbol{\zeta})-F^{-}(\boldsymbol{\zeta})=\chi^{-}(\boldsymbol{\zeta}) \frac{1}{\rho(\zeta)}, \quad \zeta \in L
$$

где мы положили $\chi(\mathbf{z})=-R_{n}(z) \psi_{n}(z) w(\mathbf{z}), \mathbf{z} \in D^{(2)}$. С помошью мероморфного абелева дифференциала (13) функция $F(\mathbf{z})$ восстанавливается по граничному условию (29) интегралом типа Коши:

$$
F(\mathbf{z})=-\frac{1}{2 \pi i} \int_{L^{+}} \chi^{-}(\boldsymbol{\zeta}) \frac{1}{\rho(\zeta)} d \Omega(\mathbf{z} ; \zeta)-p_{n}(z), \quad \mathbf{z} \notin L
$$

где $p_{n}(z) \in \mathbb{C}[z], \operatorname{deg} p_{n} \leqslant g$. Получили сингулярное интегральное уравнение для функции остатка $R_{n}$.

2. Рассмотрим это уравнение для $\mathbf{z} \in D^{(1)}$. Так как $\rho(\zeta)$ голоморфина на каждой связной компоненте $E_{j}$ компакта $E$, то $\rho(\zeta)$ голоморфна на каждой кривой $L_{j}$. Поэтому контур $L$ можно, не меняя значения интеграла, покомпонентно продеформировать в близкий контур $L^{\prime}$, также состоящий из $g+1$ компоненты и целиком расположенный на втором листе $D^{(2)}$ в некоторой окрестности $L$, в которой определена и голоморфна функция $\rho(\zeta)$ (напомним, что функция $\chi(\zeta)$ определена и голоморфна на всем втором листе $\left.D^{(2)}\right)$. Полученная интегральная формула задает аналитическое продолжение функции $F(\mathbf{z}), \mathbf{z} \in D^{(1)}$, через контур $L$ на второй лист римановой поверхности вплоть до контура $L^{\prime}$ (для $\mathbf{z} \in D^{(2)}$ оно, вообще говоря, не совпадает с функцией $F(\mathbf{z}), \mathbf{z} \in D^{(2)}$, определенной выше). Отсюда, учитывая определения функций $F$ и $\chi$, получаем:

$$
\begin{aligned}
\left(R_{n} \varphi_{n} w\right)(z) & =\frac{1}{2 \pi i} \int_{L^{\prime}}\left(R_{n} \psi_{n} w\right)(\zeta) \frac{1}{\rho(\zeta)} d \Omega\left(z^{(1)} ; \zeta^{(2)}\right)-p_{n}(z) \\
& =\frac{1}{2 \pi i} \int_{L^{\prime}}\left(R_{n} \varphi_{n} w\right)(\zeta) \frac{\psi_{n}(\zeta)}{\varphi_{n}(\zeta)} \frac{1}{\rho(\zeta)} d \Omega\left(z^{(1)} ; \zeta^{(2)}\right)-p_{n}(z), z \in \bar{D}
\end{aligned}
$$

Выберем теперь и зафиксируем произвольное достаточно малое $\varepsilon>0$, удовлетворяющее следующим условиям: линия уровня функции Грина $L(\varepsilon)=\{z$ : $\left.g_{D}(z, \infty)=\varepsilon\right\}$ состоит ровно из $g+1$ компоненты; функция $\rho$ голоморфна внутри и на $L(\varepsilon)$; для достаточно больших $n \in \Lambda$ все точки $z_{j}(n) \in D(\varepsilon)$, где $D(\varepsilon)=\left\{z: g_{D}(z, \infty)>\varepsilon\right\}$. Только такие $n \in \Lambda$ мы и рассматриваем в дальнейшем. Последовательность $\left\{\varepsilon_{n}\right\}_{n \in \Lambda}, \varepsilon_{n} \in[\varepsilon / 2,3 \varepsilon / 4]$, выберем, исходя из следующего условия:

$$
\max _{z \in L\left(\varepsilon_{n}\right)}\left|p_{n}(z)\right|=O(1) \cdot \min _{z \in L\left(\varepsilon_{n}\right)}\left|p_{n}(z)\right|
$$

при $n \rightarrow \infty, n \in \Lambda$ (это, очевидно, возможно, так как $\operatorname{deg} p_{n} \leqslant g$ ).

Пусть теперь контур $L^{\prime}=L^{\prime}(n) \subset D^{(2)}$ в (31) таков, что в области $D$ ему соответствует линия уровня $L\left(\varepsilon_{n}\right)$. Подчеркнем еше раз, что формула $(31)$ справедлива в замкнутой области $\overline{D^{(1)}}$, т.е. остается верной и для $z=z^{(1)} \in L$ : непосредственно из явного вида (15) дифференциала $d \Omega\left(z^{(1)} ; \zeta^{(2)}\right)$ вытекает, что он не имеет особенности при $z=\zeta$. 
С учетом того, что голоморфная в $\mathbb{C} \backslash E$ функция $\left(R_{n} \varphi_{n} w\right)(z)$ имеет полюс в точке $z=\infty$ порядка $\leqslant g$, и определения функций $\varphi_{n}$ и $\psi_{n}$ получаем из (31) следуюшее соотношение для максимума модуля $M_{n}$ функции $\left(R_{n} \varphi_{n} w\right)(z)$ на $E^{+} \cup E^{-}$:

$$
M_{n}=M_{n} \cdot O\left(q^{n}\right)+\max _{z \in E}\left|p_{n}(z)\right|, \quad n \in \Lambda, \quad n \rightarrow \infty
$$

$q=\exp \left\{-2 \varepsilon_{n}\right\} \in(0,1)$. Следовательно, $M_{n}=\max _{z \in E}\left|p_{n}(z)\right|\left(1+O\left(q^{n}\right)\right), n \in \Lambda$, $n \rightarrow \infty$, а значит, $\max _{z \in E}\left|\left(R_{n} \varphi_{n} w\right)(z)-p_{n}(z)\right|=M_{n} \cdot O\left(q^{n}\right)$. Из последнего соотношения и теоремы Руше вытекает, что в $D(\varepsilon)$ полином $p_{n}$ имеет $\varkappa \leqslant g$ нулей, асимптотически близких к $\varkappa$ "свободным" нулям $\varphi_{n}$.

Рассмотрим теперь интегральное уравнение $(30)$ для $\mathbf{z} \in \overline{D^{(2)}(\varepsilon)}=\left\{\mathbf{z} \in D^{(2)}\right.$ : $\left.g_{D}(z, \infty) \geqslant \varepsilon\right\}$ :

$$
\begin{aligned}
\left(-Q_{n} \psi_{n}\right)(z) & =\frac{1}{2 \pi i} \int_{L^{+}}\left(R_{n} \psi_{n} w\right)(\zeta) \frac{1}{\rho(\zeta)} d \Omega\left(z^{(2)} ; \zeta^{(2)}\right)-p_{n}(z) \\
& =\frac{1}{2 \pi i} \int_{L^{+}}\left(R_{n} \varphi_{n} w\right)(\zeta) \frac{\psi_{n}(\zeta)}{\varphi_{n}(\zeta)} \frac{1}{\rho(\zeta)} d \Omega\left(z^{(2)} ; \zeta^{(2)}\right)-p_{n}(z), z \in \overline{D(\varepsilon)}
\end{aligned}
$$

Из (32) и условия выбора $\varepsilon_{n}$ вытекает, что

$$
\max _{z \in L\left(\varepsilon_{n}\right)}\left|\left(Q_{n} \psi_{n}\right)(z)-p_{n}(z)\right|=\max _{z \in L\left(\varepsilon_{n}\right)}\left|p_{n}(z)\right| \cdot O\left(q^{n}\right)=\min _{z \in L\left(\varepsilon_{n}\right)}\left|p_{n}(z)\right| \cdot O\left(q^{n}\right),
$$

где $0<q<1$. Поскольку мы заранее выбрали $\varepsilon>0$ так, что все $g-\varkappa$ "свободных" нулей функции $\psi_{n}$ компактно (при $n \rightarrow \infty, n \in \Lambda$ ) принадлежат области $D(\varepsilon)$, то вновь с помощью теоремы Руше получаем из (33), что функции $Q_{n} \psi_{n}$ и $p_{n}$ для всех достаточно больших $n \in \Lambda$ имеют в $D\left(\varepsilon_{n}\right)$ одинаковое количество нулей. Так как функция $\psi_{n}$ имеет в конечной плоскости $g-\varkappa$ нулей, получаем, что $p_{n}$ имеет $g-\varkappa$ нулей, асимптотически близких к "свободным" нулям $\psi_{n}$. По условию теоремы $d(n)$ неспециальный при всех $n$. Следовательно, $z_{j}(n) \neq z_{k}(n)$ при $j \leqslant \varkappa$ и $k \geqslant \varkappa+1$. Значит, $\operatorname{deg} p_{n}=g$ и все его $g$ нулей компактно (по $n \in \Lambda$ ) принадлежат $D(\varepsilon)$. Отсюда уже вытекает: $\operatorname{deg} Q_{n}=n$, т.е. индекс $n$ нормальный.

Вернемся теперь к случаю $\mathbf{z} \in D^{(1)}$. Непосредственно из (31) получаем, что функция $R_{n} \varphi_{n} w$ имеет в $D_{\varepsilon}$ ровно $g$ конечных нулей. Следовательно, функция $R_{n}$ имеет в $D_{\varepsilon}$ ровно $g-\varkappa$ конечных нулей. Тем самым, для $n \in \Lambda$ аппроксимации Паде $[n / n]_{\widehat{\rho}}$ интерполируют функцию $\widehat{\rho}(z)$ дополнительно в $g-\varkappa$ точках, $\varkappa=\varkappa(n)$, компактно по $n \in \Lambda$ принадлежащих $D$.

3. В случае, когда в условиях теоремы 3 функция $f=\widehat{\rho}+r$, де $r \not \equiv 0$, необходимо по аналогии с $[4]$ и $[5 ; \S \S 3,4]$ рассмотреть модифицированную краевую задачу для кусочно мероморфной в $\Re \backslash L$ функции $\Psi_{n}(\boldsymbol{\zeta})$. Отметим, что в этой модифицированной постановке краевое условие 2) полностью сохраняется, а условие 1) на дивизор функции $\left(\Psi_{n}\right)$ в $\mathfrak{R} \backslash L$ изменяется следующим образом. К правой части этого условия добавляется дивизор нулевой степени, зависящий от $n$ и определяемый рациональной функцией $r$, точнее - ее полюсами. В остальном приведенные выше рассуждения, основанные на использовании сингулярного интегрального уравнения, полностью сохраняются.

Отметим, что в условиях теоремы 3 удается показать, что все предельные точки нулей $Q_{n}$ и $R_{n}$ описываются в терминах решений системы $(*)$, пока только для случая, когда все $z_{j}^{0}$ лежат в $g$ различных лакунах (одна из них может быть и внешней). 
СлЕДСТВИЕ 4. Если в условиях теоремы 3 при всех $n \in \Lambda \subset \mathbb{N}$ в дивизоре $d(n)=\mathbf{z}_{1}(n)+\cdots+\mathbf{z}_{g}(n)$ ровно $k, 0 \leqslant k \leqslant g$, точек $\mathbf{z}_{j}(n)$ компактно по $n$ принадлежат $D^{(2)}$, а остальнье компактно принадлежат $D^{(1)}$, то по этой подпоследовательности $\Lambda$ нули $Q_{n}$ имеют в точности $k$ предельных точек вне $E$, а свободные нули функиии $R_{n}$ имеют ровно $g-k$ предельных точек вне $E$.

СлЕДСТВИЕ 5. Если в условиях теоремы 3 величины $\omega_{1}(\infty), \ldots, \omega_{g+1}(\infty)$ рационально независимы, то любой дивизор $\operatorname{pr} d$, где $d \in \mathbb{D}$, есть предельная точка последовательности дивизоров степени $g$, составленных из нулей произведения $Q_{n} R_{n}$, располохсенных в $\mathbb{C} \backslash E$.

СлЕДСТВИЕ 6 . Пусть $g=1, f_{1}=\widehat{\rho}_{1}+r_{1}$, әде допустимый вес $\rho_{1}$ марковский, $r_{1} \in \mathbb{C}(z) \cap \mathscr{H}(E)$, a $f_{2}=\widehat{1 / p}$, где $p \in \mathbb{C}[z]$ u $p(z)=\prod_{j=1}^{m}\left(z-\overline{b_{j}}\right)^{2}$, $b_{1}, \ldots, b_{m} \in D-$ полюсы $r_{1}$. Тогда мнодество pr $\mathfrak{D}^{(2)}$ (см. п. $6 \S 3$ ) для голоморфной в $D$ функиии $f_{2}$ совпадает с кривой $\gamma$ из теоремы Рахманова для мероморфной в $D$ функиии $f_{1}$.

Отметим, что $\gamma=\operatorname{pr} \gamma^{(2)}\left(f_{1}\right)$, где $\gamma^{(2)}\left(f_{1}\right)=\mathfrak{D}^{(2)}\left(f_{1}\right)$ "невидимая" часть ортогональной траектории некоторого квадратичного дифференциала на $\mathfrak{R}$, а на проекции другой части части $\gamma^{(1)}\left(f_{1}\right)=\mathfrak{D}^{(1)}\left(f_{2}\right)$ этой ортогональной траектории расположены все предельные точки свободных нулей функции остатка $R_{n}$.

ДоКАЗАТЕЛЬСТво СЛЕДСТВИЯ 6. Поскольку вес $\rho_{1}$ марковский, из п. $5 \S 2$ вытекает, что $x$-координаты вектора $\xi^{0}=\xi^{0}\left(f_{1}\right)$ полностью определяются величинами $\Omega_{k}\left(a^{(2)}\right)$. Рассмотрим две функции $f_{1}=\widehat{\rho}_{1}+r_{1}$ и $f_{2}=\widehat{1 / p}$, где $p(z)=\prod_{j=1}^{m}\left(z-\overline{b_{j}}\right)^{2}$, а $b_{1}, \ldots, b_{m} \in D$, как и прежде, - полюсы $r$; тем самым, $\operatorname{deg} p=2 m$. Результаты п. $5 \oint 2$ с учетом того, что $\Omega_{k}\left(z^{(1)}\right)-\Omega_{k}\left(\bar{z}^{(2)}\right) \in i \mathbb{R}$ $(\bmod$ периодов), показывают, что при таком выборе полинома $p \in \mathbb{C}[z]$ имеем: $x_{k}^{0}\left(f_{1}\right)=x_{k}^{0}\left(f_{2}\right)$, т.е. $x$-координаты начальных условий совпадают, тем самым совпадают и соответствующие множества $\mathbb{D}=\mathbb{D}\left(x^{0}\right)$. Следовательно, в случае одной лакуны (т.е. при $g=1$ ) получаем, что множество $\operatorname{pr} \mathfrak{D}^{(2)}$ - дуга $\gamma=\gamma\left(f_{2}\right)$, совпадающая с дугой $\gamma=\gamma\left(f_{1}\right)$, возникаюшей по теореме С. Тем самым, (4) имеет место и для голоморфной в $D$ функции $f=f_{2}$.

\section{§5. Случай рода $g=2$}

При $g=2$ всякий специальный дивизор $d$ имеет вид $d=z^{(1)}+z^{(2)}$. Для такого дивизора $\Omega_{k}\left(z^{(1)}\right)+\Omega_{k}\left(z^{(2)}\right) \equiv 0$, т.е. его $x$-, $y$-координаты равны нулю. Следовательно, если мы выберем начальные условия $d^{0}$ так, что $x_{1}^{0} \neq 0$ или $x_{2}^{0} \neq 0$, то и $d^{0}$, и всякий дивизор, принадлежащий $\mathbb{D}\left(x^{0}\right)$, будет неспециальным.

Изучим теперь строение множества $\mathbb{D}\left(x^{0}\right)=\left\{d=\mathbf{z}+\boldsymbol{\zeta}: \operatorname{Re} \Omega_{k}(\mathbf{z})+\operatorname{Re} \Omega_{k}(\boldsymbol{\zeta})=\right.$ $\left.x_{k}^{0}(\bmod 1)\right\}$ более детально. Нетрудно видеть, что точка $z \in E_{1}^{\prime}$ тогда и только тогда, когда $\operatorname{Re} \Omega_{1}(\mathbf{z})=1 / 2(\bmod 1), \operatorname{Re} \Omega_{2}(\mathbf{z})=0(\bmod 1)$. Аналогично, $\zeta \in E_{2}^{\prime}$ тогда и только тогда, когда $\operatorname{Re} \Omega_{k}(\zeta)=1 / 2(\bmod 1), k=1,2$. Если при некотором $x^{0}$ множество $\mathbb{D}\left(x^{0}\right)$ содержит дивизор $d=\mathbf{z}+\zeta$ с $z \in E_{1}^{\prime}$, то для $\boldsymbol{\zeta}$ имеем:

$$
\operatorname{Re} \Omega_{1}(\boldsymbol{\zeta})=x_{1}^{0}-\frac{1}{2} \quad(\bmod 1), \quad \operatorname{Re} \Omega_{2}(\boldsymbol{\zeta})=x_{2}^{0} \quad(\bmod 1)
$$

Если мы выбрали $x^{0}$ так, что $\zeta \notin E_{2}^{\prime}$, то, тем самьм, точка $\zeta$ лежит на пересечении соответствующих ортогональных траекторий квадратичных дифференциалов $d \Omega_{1}^{2}$ 
и $d \Omega_{2}^{2}$ (в дальнейшем в этом параграфе мы будем говорить короче: траектории дифференциалов $\left.d \Omega_{k}\right)$. Нетрудно видеть, что если $t_{k} \in(-1 / 2,1 / 2), t_{k} \neq 0$, то две траектории $\operatorname{Re} \Omega_{k}(\boldsymbol{\zeta})=t_{k}$ и $\operatorname{Re} \Omega_{k}(\boldsymbol{\zeta})=-t_{k}$ лежат одна над другой на разных листах $\mathfrak{R}$ и, тем самьм, не пересекаются (здесь $k=1,2)$. Если же мы возьмем $t_{1} \in(-1 / 2,0), t_{2} \in(0,1 / 2)$, то не пересекаются траектории $\operatorname{Re} \Omega_{1}(\boldsymbol{\zeta})=t_{1}$ и $\operatorname{Re} \Omega_{2}(\boldsymbol{\zeta})=t_{2}$. Следовательно, при $x_{1}^{0}, x_{2}^{0} \in(0,1 / 2)$ соотношения $(34)$ вьполняться не могут. Аналогично анализируется и ситуация, когда $\zeta \in E_{2}^{\prime}$ или $\zeta \in E_{3}^{\prime}$. В итоге оказывается, что найдутся координаты $x_{1}^{0}, x_{2}^{0} \in(0,1 / 2)$ (при этом $x_{1}^{0}$ близко к $0, \mathrm{a}$ $\left.x_{2}^{0}-\mathrm{k} 1 / 2\right)$ такие, что для некоторых $d=\mathbf{z}+\zeta \in \mathbb{D}\left(x^{0}\right)$ имеем: $z, \zeta \notin E_{1}^{\prime} \cup E_{2}^{\prime} \cup E_{3}^{\prime}$. Таким образом, для таких $x^{0}$ множество $\mathbb{D}\left(x^{0}\right) \neq S^{g} \mathfrak{R}$.

Итак, пусть начальные условия $d^{0}$ таковы, что для $d \in \mathbb{D}\left(x^{0}\right)$ имеем: $z, \zeta \notin E_{1}^{\prime} \cup$ $E_{2}^{\prime} \cup E_{3}^{\prime}$. Нетрудно видеть, что найдется $d \in \mathbb{D}\left(x^{0}\right)$ такой, что $z, \zeta \notin \mathbb{R}$. Через точку $\mathbf{z}$ проходит ровно по одной траектории $\gamma_{1}$ и $\gamma_{2}$ дифференциалов $d \Omega_{1}$ и $d \Omega_{2}$. Взаимное расположение этих кривых описывается следующим образом.

Лемма 2. Пусть $\varphi-$ угол пересечения траекторий $\gamma_{1} u \gamma_{2}$. Тогда $\varphi \neq 0$ $(\bmod \pi)$.

ДОКАЗАТЕЛЬСТво. Пусть $\mathbf{z}_{1}=\psi_{1}(t)$ и $\mathbf{z}_{2}=\psi_{2}(t)$ - уравнения траекторий $\gamma_{1}$ и $\gamma_{2}$ соответственно, т.е. $\Omega_{1}\left(\mathbf{z}_{1}\right)-\Omega_{1}(\mathbf{z})=i t$ и $\Omega_{2}\left(\mathbf{z}_{2}\right)-\Omega_{2}(\mathbf{z})=i t, t \in \mathbb{R}$. Дифференцируя эти соотношения, получаем $\psi_{j}^{\prime}(t) \Omega_{j}^{\prime}\left(\mathbf{z}_{j}\right)=i$ при $j=1,2$. Если бы кривые $\gamma_{1}$ и $\gamma_{2}$ касались друг друга в точке $\mathbf{z}$, то в представлении $\psi_{j}^{\prime}(0)=R_{j} e^{i \theta_{j}}$ мы бы имели $\theta_{1}=\theta_{2}=\theta(\bmod \pi)$, т.е. $\psi_{1}^{\prime}(0) / \psi_{2}^{\prime}(0) \in \mathbb{R}$. Откуда получили бы, что $\Omega_{1}^{\prime}(\mathbf{z}) / \Omega_{2}^{\prime}(\mathbf{z}) \in \mathbb{R}$. Поскольку справедливо представление $\Omega_{j}^{\prime}(\mathbf{z})=\left(a_{j} z+b_{j}\right) / w(\mathbf{z})$, где $a_{j}, b_{j} \in i \mathbb{R}$, то для точки $z \mathrm{c} \operatorname{Im} z \neq 0$ имеем: $a_{1} z+b_{1}=\lambda\left(a_{2} z+b_{2}\right)$, где $\lambda \in \mathbb{R}$. Тем самым, $a_{1}=\lambda a_{2}, b_{1}=\lambda b_{2}$, чего быть не может, так как дифференциалы $d \Omega_{1}$ и $d \Omega_{2}$ образуют базис. Непосредственно из доказательства вытекает, что $\varphi \neq \pi / 2$ $(\bmod \pi)$.

Таким образом, взаимное расположение траекторий дифференциалов $d \Omega_{1}$ и $d \Omega_{2}$ в некоторых окрестностях $U$ и $V$ точек $\mathbf{z}$ и $\boldsymbol{\zeta}$ для выбранного $d=\mathbf{z}+\boldsymbol{\zeta} \in \mathbb{D}\left(x^{0}\right)$ таково, что они образуют (локальную) систему “криволинейных координат" (см. рис. 1) в следуюшем смысле. Любые две траектории $d \Omega_{1}$ и $d \Omega_{2}$, проходящие через окрестность $U$, пересекаются между собой и притом ровно в одной точке; аналогичное справедливо и для $V$.
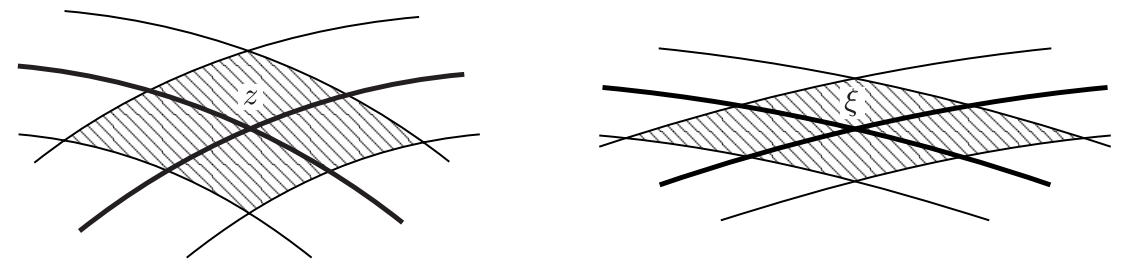

Рис. 1. Взаимное расположение ортогональных траекторий квадратичных диффееренциалов $d \Omega_{1}^{2}$ и $d \Omega_{2}^{2}$ в окрестностях точек $z$ и $\zeta$

Пусть $\operatorname{Re} \Omega_{j}(\mathbf{z})=\alpha_{j}, \operatorname{Re} \Omega_{j}(\boldsymbol{\zeta})=\beta_{j}$, где $\alpha_{j}, \beta_{j} \in(-1 / 2,1 / 2)$ и $\alpha_{j}+\beta_{j}=x_{j}^{0}$ $(\bmod 1), j=1,2$. Пусть $\varepsilon>0$ таково, что $\alpha_{j} \pm \varepsilon, \beta_{j} \pm \varepsilon \in(-1 / 2,1 / 2)$. Определим 
$\varepsilon$-окрестности $U_{\varepsilon} \subset U$ и $V_{\varepsilon} \subset V$ для точек $\mathbf{z}$ и $\boldsymbol{\zeta}$ соответственно следуюшим образом. Окрестность $U_{\varepsilon}$ состоит из точек $\mathbf{z}^{\prime}$, для которых $\operatorname{Re} \Omega_{j}\left(\mathbf{z}^{\prime}\right)=\alpha_{j}+t_{j}$, $t_{j} \in(-\varepsilon, \varepsilon)$. Аналогично определяется окрестность $V_{\varepsilon}$ точки $\boldsymbol{\zeta}$. Нетрудно видеть, что любой дивизор $d^{\prime}=\mathbf{z}^{\prime}+\boldsymbol{\zeta}^{\prime}$, в котором точки $\mathbf{z}^{\prime}$ и $\boldsymbol{\zeta}^{\prime}$ являются решением системы уравнений

$$
\begin{array}{ll}
\operatorname{Re} \Omega_{1}\left(\mathbf{z}^{\prime}\right)=\alpha_{1}+t_{1}, & \operatorname{Re} \Omega_{2}\left(\mathbf{z}^{\prime}\right)=\alpha_{2}+t_{2}, \\
\operatorname{Re} \Omega_{1}\left(\boldsymbol{\zeta}^{\prime}\right)=\beta_{1}-t_{1}, & \operatorname{Re} \Omega_{2}\left(\boldsymbol{\zeta}^{\prime}\right)=\beta_{2}-t_{2}, \quad \text { где } t_{1}, t_{2} \in(-\varepsilon, \varepsilon),
\end{array}
$$

принадлежит множеству $\mathbb{D}\left(x^{0}\right)$. Таким образом, множество pr $\mathfrak{D}$ содержит открытое множество $\operatorname{pr} U_{\varepsilon} \sqcup \operatorname{pr} V_{\varepsilon}$, т.е. является “двумерным".

\section{Список литературы}

1. Чебьиёв П. Л. О непрерьвных дробях // Ученые записки Имп. академии наук. 1855. Т. III. С. 636-664. (Полное собрание сочинений. Т. II. М.-Л.: Изд-во АН СССР, 1948. C. 103-126.)

2. Markoff A.A. Deux démonstrations de la convergence de certaines fractions continues // Acta Math. 1895. V. 19. Р. 93-104. (Марков А.А. Два доказательства сходимости некоторых непрерывных дробей // Избранњые труды по теории непрерывных дробей и теории функций, наименее уклоняющихся от нуля. М.: Гостехиздат, 1948. С. 106-119.)

3. Марков A.A. О корнях некоторых уравнений. I // Избранные труды по теории непрерывных дробей и теории функций, наименее уклоняющихся от нуля. М.: Гостехиздат, 1948. С. 34-43.

4. Суетин С. П. О равномерной сходимости диагоналњыых аппроксимаций Паде для гиперэллиптических функций // Матем. сб. 2000. Т. 191. №9. С. 81-114.

5. Суетин С. П. Аппроксимации Паде и эффективное аналитическое продолжение степенного ряда // УМН. 2002. Т. 57. № 1. С. 45-142.

6. Гончар A. A. О сходимости аппроксимаций Паде для некоторых классов мероморфыных функций // Матем. сб. 1975. Т. 97 (139). С. 607-629.

7. Рахманов E. A. О сходимости диагональных аппроксимаций Паде // Матем. сб. 1977. T. 104 (146). № 2. C. 271-291.

8. Peherstorfer F. Zeros of polynomials orthogonal on several intervals // arXiv: math-ph/ 0203058 v1. 28 Mar 2002.

9. Гончар A. A. О равномерной сходимости диагональных аппроксимаций Паде // Матем. сб. 1982. Т. 118 (160). № 4 (8). С. 535-556.

10. Stahl $H$. Orthogonal polynomials with complex valued weight function. I, II // Constr. Approx. 1986. V. 2. P. 225-240; 241-251.

11. Stahl H. Diagonal Padé approximants to hyperelliptic functions // Ann. Fac. Sci. Toulouse Math. (6). 1996. Spec. Iss. P. 121-193.

12. Бейкер Дж., Грейвс-Моррис П. Аппроксимации Паде. М.: Мир, 1986.

13. Stahl H. Conjectures around Baker-Gammel-Wills conjecture // Constr. Approx. 1997. V. 13. P. 287-292.

14. Buslaev V.I., Simple counterexample to the Baker-Gammel-Wills conjecture // East J. Approx. 2001. V. 7. № 4. P. 515-517.

15. Буслаев В. И. О гипотезе Бейкера-Гаммеля-Уиллса в теории аппроксимаций Паде // Матем. сб. 2002. Т. 193. №6. С. 25-38.

16. Бернштейн C. Н. О многочленах, ортогональных на конечном отрезке // Собрание сочинений. Т. II. М.: АН СССР, 1952. С. 7-106.

17. Голузин Г. М. Геометрическая теория функций комплексного переменного. М.: Наука, 1966.

18. Суетин С. П. О динамике "блуждающих" нулей полиномов, ортогоналњных на нескольких отрезках // УМН. 2002. Т. 57. № 2. С. 199-200.

19. Ахиезер Н. И. Об ортогональных многочленах на нескольких интервалах // Докл. АН CCCP. 1960. Т. 134. № 1. С. 9-12. 
20. Ахиезер Н.И., Томчук Ю.Я. К теории ортогоналњных многочленов на нескольких интервалах // Докл. АН СССР. 1961. Т. 138. № 4. С. 743-746.

21. Ахиезер Н. И. Континуалњые аналоги ортогоналњных многочленов на системе интервалов // Докл. АН СССР. 1961. Т. 141. № 2. С. 263-266.

22. Nuttall J., Singh R.S. Orthogonal polynomials and Padé approximants associated with a system of arcs // J. Approx. Theory. 1977. V. 21. P. 1-42.

23. Nuttall J. Asymptotics of diagonal Hermite-Padé polynomials // J. Approx. Theory. 1984. V. 42. P. 299-386.

24. Widom $H$. Extremal polynomials associated with a system of curves in the complex plane // Adv. Math. 1969. V. 3. P. 127-232.

25. Аптекарев А.И. Асимптотические свойства многочленов, ортогональных на системе контуров, и периодические движения цепочек Тода // Матем. сб. 1984. Т. 125 (167). № 2 (10). C. 231-258.

26. Deift P., Kriecherbauer T., McLaughlin K.T-R., Venakides S., Zhou X. Uniform asymptotics for polynomials orthogonal with respect to varying exponential weights and applications to universality questions in random matrix theory // Preprint, 1998.

27. Chen Y., Lawrence N. A generalisation of the Chebyshev polynomials // Preprint, 2001.

28. Dumas $S$. Sur le développement des fonctions elliptiques en fractions continues // Thesis. Zürich, 1908.

29. Никишин E. M. О сходимости диагональных аппроксимаций Паде для некоторых функций // Матем. сб. 1976. Т. 101 (143). № 2. С. 280-292.

30. Зверович Э.И. Краевые задачи теории аналитических функций в гёльдеровских классах на римановых поверхностях // УМН. 1971. Т. 26. № 1. С. 113-180.

31. Форстер О. Римановы поверхности. М.: Мир, 1980.

32. Спрингер Джс. Введение в теорию римановых поверхностей. М.: ИЛ, 1960.

33. Дубровин Б. А. Тэта-функции и нелинейные уравнения // УМН. 1981. Т. 36. № 2 . C. $11-80$.

34. Krazer A. Lehrbuch der Thetafunktionen. New York: Chelsea Publishing Company, XXIV, 1970.

35. Джсенкинс Джс. Однолистные функции и конформные отображения. М.: ИЛ, 1962.

36. Арнольд В. И. Математические методы классической механики. М.: Наука, 1989.

37. Дубровин Б. А. Периодические задачи для уравнения Кортевега-де Фриза в классе конечнозонных потенциалов // Функц. анализ и его прилож. 1975. Т. 9. № 3. С. 41-52.

38. Левитан Б. М. Обратные задачи Штурма-Лиувилля. М.: Наука, 1984.

39. Рахманов E. A. Некоторые вопросы сходимости диагональных аппроксимаций Паде // Дисс. ... докт. физ.-матем. наук. М.: МИАН, 1983.

40. Максвелл Джс. К. Трактат об электричестве и магнетизме. Т. І. М.: Наука, 1989.

41. IIиффер $M$. Некоторые новые результаты в теории конформных отображений // Приложение к книге Р. Куранта "Принцип Дирихле, конформные отображения и минимальные поверхности". М.: ИЛ, 1953. С. 234-301.

Математический институт

им. В.А. Стеклова РАН

E-mail: suetin@mi.ras.ru
Поступила в редакцию 21.01 .2002 и 14.10 .2002 